\title{
INVESTIGATION OF BRIDGE SCOUR AT SELECTED SITES ON MISSOURI STREAMS
}

\author{
U.S. GEOLOGICAL SURVEY
}

Water-Resources Investigations Report 94-4200

GRAND RIVER NR SUMNER, MO.

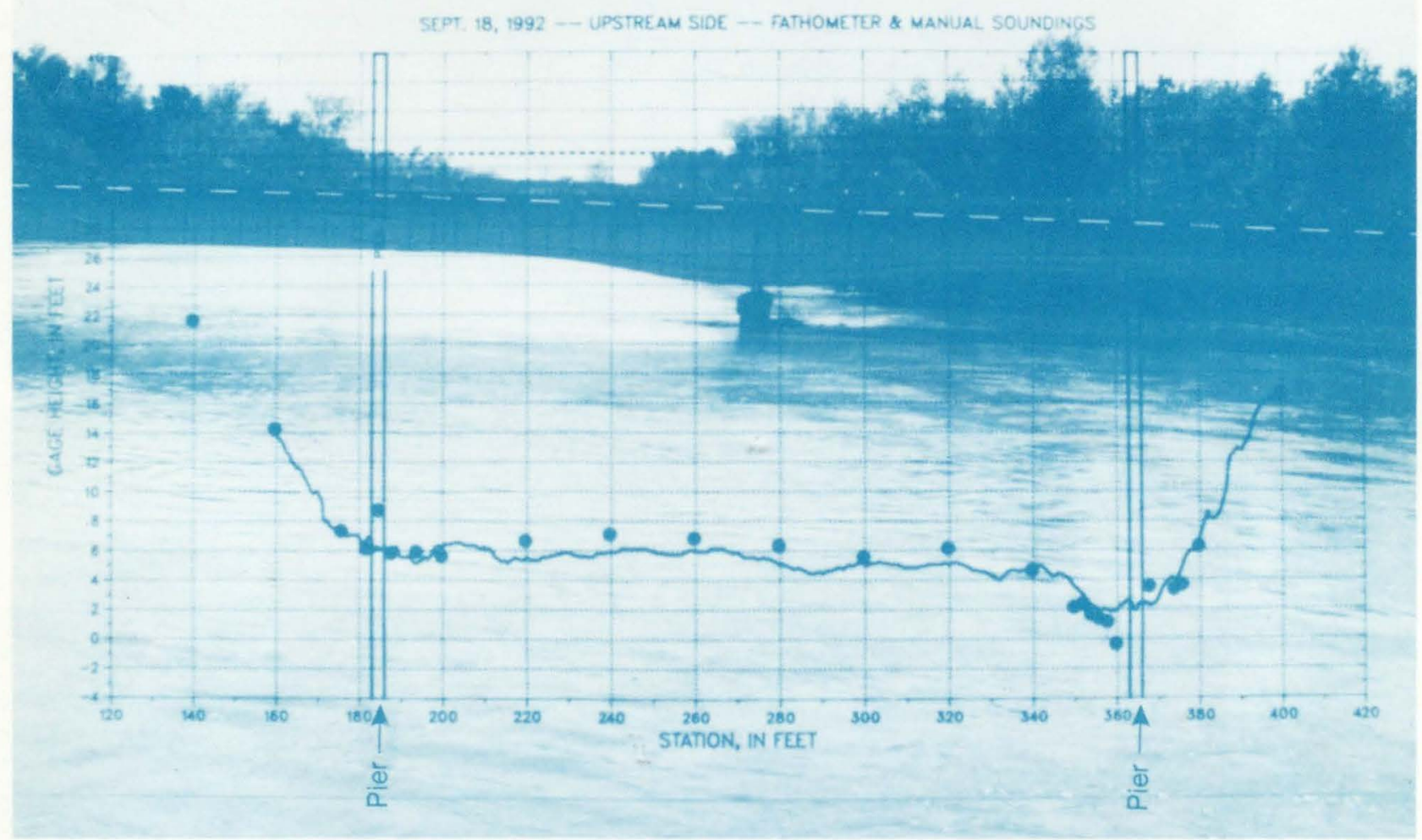

Prepared in cooperation with the

MISSOURI HIGHWAY AND TRANSPORTATION COMMISSION

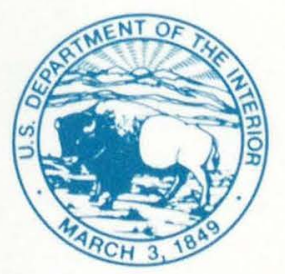


Cover photograph. View of the Grand River near Sumner, Missouri, September 18, 1992, and graphical chart showing streambed configuration near two bridge piers. 


\section{INVESTIGATION OF BRIDGE SCOUR AT SELECTED SITES ON MISSOURI STREAMS}

BY LAWRENCE D. BECKER

U.S. GEOLOGICAL SURVEY

Water-Resources Investigations Report 94-4200

U.S. GEOLOGICAL SURVEY RESTON, VA.

JAN 271995

LI B R A R Y

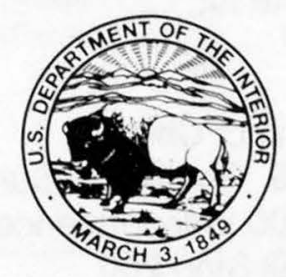




\title{
U.S. DEPARTMENT OF THE INTERIOR \\ BRUCE BABBITT, Secretary
}

\author{
U.S. GEOLOGICAL SURVEY
}

GORDON P. EATON, Director

For additional information write to:

District Chief

U.S. Geological Survey

1400 Independence Road

Mail Stop 200

Rolla, Missouri 65401
Copies of this report may be purchased from:

U.S. Geological Survey Earth Science Information Center Open-File Reports Section

Box 25286, MS 517

Federal Center

Denver, Colorado 80225 


\section{CONTENTS}

Abstract

Introduction

Purpose and Scope

Acknowledgments...

Methods of Study of Bridge Scour.

Description of Study Sites

Measured Scour Depths..

Estimated Scour Depths and Selected Scour Equations.......

Measured and Estimated Scour Depths.

An Example of a Scour-Related Bridge Failure

Summary

Selected References.

\section{FIGURES}

1. Map showing location of study sites and physiographic regions in Missouri

2-8. Photographs showing:

2. View of downstream side of bridge at time of scour measurement made during the flood of September 28, 1993, at the Gasconade River near Rich Fountain, Missouri.....

3. Pileup of water surface at upstream side of bridge pier during the flood of May 13, 1991, at Little Piney Creek at Newburg, Missouri

4. Turbulent flow at and downstream from bridge pier during the flood of May 13, 1991, at Little Piney Creek at Newburg, Missouri.....

5. Floating woody debris during the flood crest on April 21, 1991, at the Grand River near Sumner, Missouri

6. Woody debris accumulated against the center bridge pier partially blocking the bridge opening on the upstream side during the flood of July 8, 1993, at the Chariton River near Prairie Hill, Missouri..

7. View of the downstream side of bridge and woody debris accumulated on the center pier extending completely through the bridge opening during the flood of July 8, 1993, at the Chariton River near Prairie Hill, Missouri.

8. Woody debris accumulated on upstream side of bridge piers partially blocking the bridge opening following the flood of April 12, 1992, at the Chariton River at Novinger, Missouri

9-11. Graphs showing:

9. Streambed cross sections at the Chariton River near Prairie Hill, Missouri

10. Effect of angle of attack on bridge pier.

11. Measured scour depths for each of the selected sites in Missouri 
12. Relation between mean bed-material diameter and measured scour depth at selected sites in Missouri

13. Relation between flow depth and measured scour depth at selected sites in Missouri

14. Relation between pier width and measured scour depth at selected sites in Missouri

15. Relation between flow velocity and measured scour depth at selected sites in Missouri

16. Boxplots of the distribution of residuals of estimated scour depths (using scour equations)

from measured scour depths at selected sites in Missouri.....

17-23. Graphs showing:

17. Relation between scour depth estimated using the Laursen equation and the residual at selected sites in Missouri....

18. Relation between scour depth estimated using the Larras equation and the residual at selected sites in Missouri.

19. Relation between scour depth estimated using the Chitale equation and the residual at selected sites in Missouri

20. Relation between scour depth estimated using the Inglis-Poona II equation and the residual at selected sites in Missouri....

21. Relation between scour depth estimated using the Shen equation and the residual at selected sites in Missouri.

22. Relation between scour depth estimated using the Froehlich equation and the residual at selected sites in Missouri.

23. Relation between scour depth estimated using the Colorado State University equation and the residual at selected sites in Missouri

24-26. Photographs showing:

24. View of upstream side of State Highway 32 bridge over North Cobb Creek near Lebanon, Missouri

25. Aerial view of State Highway 32 crossing at North Cobb Creek near Lebanon, Missouri, showing a scour-related bridge failure that occurred during the flood of May 26, 1990

26. The large, deep scour hole formed during the flood of May 26, 1990, on North Cobb Creek near Lebanon, Missouri

\section{TABLES}

1. Summary of discharge data at selected sites in Missouri

2. Summary of pier, scour, and hydraulic data collected at selected sites in Missouri

3. Measured scour depths and scour depths estimated using local-pier-scour equations at selected sites in Missouri

4. Pier-shape coefficients

5. Statistical characteristics of measured and estimated scour depths at selected sites in Missouri 
CONVERSION FACTORS

\begin{tabular}{rll}
\hline Multiply inch pound unit & by & To obtain metric unit \\
\hline inch (in.) & 25.4 & millimeter \\
foot (ft) & 0.3048 & meter \\
square $\mathrm{mile}\left(\mathrm{mi}^{2}\right)$ & 2.590 & square kilometer \\
foot per second $(\mathrm{ft} / \mathrm{s})$ & 0.3048 & meter per second \\
cubic foot per second $\left(\mathrm{ft}^{3} / \mathrm{s}\right)$ & 0.02832 & cubic meter per second \\
\hline
\end{tabular}





\title{
Investigation of Bridge Scour at Selected Sites on Missouri Streams
}

\author{
By Lawrence D. Becker
}

\begin{abstract}
Scour around bridge piers is a major concern in the design of a new bridge or the evaluation of the structural stability of an existing bridge. An adequate estimation of potential scour at bridge piers is essential to proper design, construction, and maintenance of hydraulic structures.

Reasonably accurate estimates of scour depth are needed for safe, cost-effective bridge design. Although many equations have been developed on the basis of laboratory work that can be used to estimate local scour at piers, site-specific data have not previously been collected to verify the accuracy of these equations or applicability to Missouri streams.

Scour data were collected during floods at 10 sites on streams in Missouri. The recurrence intervals of the floods ranged from less than 2 to more than 50 years. Local-scour holes near bridge piers ranged from 0.5 to 7.1 feet deep. Seven local-pier-scour equations were evaluated as to their usefulness in estimating the measured scour at the sites studied. None of the medians of scour depths estimated using the seven equations were statistically equal to the median of the measured scour depths at a 0.05 level of significance. However, the Froehlich equation without a safety factor provided the "best fit" estimates of the equations considered. Estimates from the Froehlich equation with a safety factor compare closely with estimates from the Colorado State University equation.
\end{abstract}

Contraction scour exceeded local scour by several times in some cases. Total scour of approximately 19 feet, largely because of contraction scour, was measured at the Chariton River near Prairie Hill during the flood of July 8, 1993. This large scour was, in part, the result of an accumulation of woody debris decreasing the bridge-opening area.

A bridge on State Highway 32 near Lebanon over North Cobb Creek (drainage area 52.5 square miles) was destroyed during the flood of May 26, 1990. Bridge scour attributable to contraction of the flood flow caused this loss.

\section{INTRODUCTION}

Scour around bridge piers is a major concern in the design of a new bridge or the evaluation of the structural stability of an existing bridge. Three types of scour can occur at a bridge: general scour, contraction scour, and local scour. General scour is the progressive degradation or lowering of the streambed through natural or human-induced processes. Channel degradation generally results from increased discharge, decreased bedload, or decreased bed-material size (Galay, 1983). Lateral erosion caused by a shift in the flow or meander pattern is included with general scour. Contraction scour is streambed erosion caused by increased flow velocity near a bridge or other channel constriction resulting from the decrease in flow area at the contracted opening, such as that caused by a bridge, approach embankments, and piers. 
Obstruction of the bridge opening by debris also results in decreased flow area and may cause or increase contraction scour at a site. Local scour is erosion caused by local disturbances in the flow, such as vortices and eddies, in the vicinity of piers (Butch, 1991).

Many local-pier-scour equations (hereafter referred to as scour equations in this report) have been developed by numerous investigators based on laboratory studies that can be used to estimate scour depths at piers. Some of the independent variables used in many of the equations are pier geometry, median bedmaterial diameter, flow depth, and velocity. Application of these equations to actual bridge sites commonly results in a considerable range of estimated scour depths. One equation may estimate little or no scour at a bridge pier and another equation may overestimate scour depth. However, site-specific data have not previously been collected to verify the accuracy of these equations or their applicability to Missouri streams.

Reasonably accurate estimates of scour depth are needed for safe, cost-effective bridge design. This need has resulted in efforts to collect scour data during floods under varied conditions. Scour depths measured during floods are a result of unique site and flow conditions that are more complex and varied than flows produced in a laboratory. In recent years, studies by several Federal and State agencies (Simon and Outlaw, 1989; Landers and Trent, 1991; Southard, 1992) have involved the collection of scour data at bridges to develop and evaluate a national data base that can be used to investigate scour processes and develop and evaluate scour prediction techniques.

The U.S. Geological Survey (USGS), in cooperation with the Missouri Highway and Transportation Department (MHTD), began a study of scour around bridge piers during 1988 under an agreement with the Missouri Highway and Transportation Commission. The objectives of this study were to collect scour data during floods and to evaluate the usefulness of available scour equations for estimating local scour.

Selected scour data collected as part of this study are available for inclusion in the USGS National Scour Data Base for future analyses on a regional or national basis.

\section{Purpose and Scope}

This report summarizes scour data collected at 10 study sites during floods on streams in Missouri (fig. 1). The methods used to collect scour data are briefly described and the bridge geometry, hydraulic characteristics, and scour measurements at each site are summarized. Data collected and presented in the report include pier type and width; median bed-material diameter; flow velocity, depth, and angle; and measured scour depths at piers. Seven existing scour equations were compared in this study. The equations were evaluated on the basis of their usefulness in estimating the measured scour at the 10 study sites. The relation between factors such as pier width, median bed-material diameter, flow velocity, and flow depth, and measured scour depths at the study sites were investigated. Scour estimates calculated using the various equations are graphically and statistically compared to the measured scour depths to evaluate applicability of equations to Missouri streams. Two specific cases of substantial contraction scour, one of which resulted in loss of a State highway bridge, also are briefly described.

\section{Acknowledgments}

Appreciation is extended to personnel of the MHTD for providing bridge plans, cross section data, photographs, and other data. Appreciation also is extended to those field personnel of the MHTD who provided assistance with debris clearance throughout the project and safety considerations (traffic control) at times when extremely hazardous working conditions existed. 


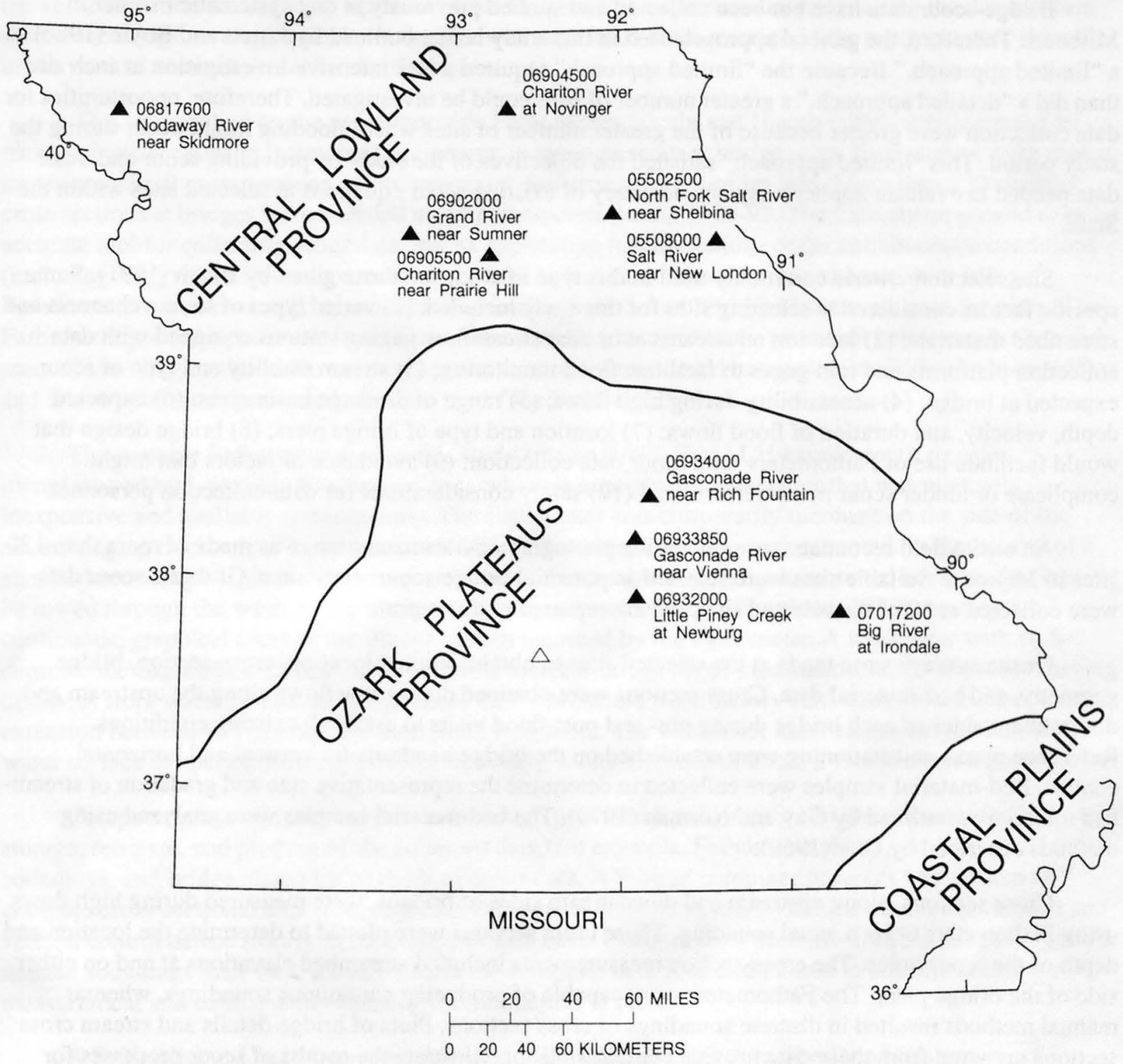

EXPLANATION

06904500

Chariton River

at Novinger $\Delta$ SCOUR STUDY SITE,

NUMBER, AND NAME

$\triangle$ NORTH COBB CREEK

NEAR LEBANON

Figure 1. Location of study sites and physiographic regions in Missouri (physiography from Fenneman, 1938). 


\section{METHODS OF STUDY OF BRIDGE SCOUR}

Bridge-scour data have not been collected and studied previously in any systematic manner in Missouri. Therefore, the general approach used in this study is that outlined by Jarrett and Boyle (1986) as a "limited approach." Because the "limited approach" required a less intensive investigation at each site than did a "detailed approach," a greater number of sites could be investigated. Therefore, opportunities for data collection were greater because of the greater number of sites where flooding might occur during the study period. This "limited approach" satisfied the objectives of the study by providing scour and other data needed to evaluate applicability and accuracy of existing scour equations at selected sites within the State.

Site selection criteria commonly used in this type of study are those given by Butch (1991). Some specific factors considered in selecting sites for this study included: (1) varied types of stream channels and streambed materials; (2) location on streams at or near streamflow gaging stations equipped with data collection platforms and rain gages to facilitate flood monitoring; (3) stream stability and type of scour expected at bridge; (4) accessibility during high flows; (5) range of drainage basin sizes; (6) expected depth, velocity, and duration of flood flows; (7) location and type of bridge piers; (8) bridge design that would facilitate use of Fathometers ${ }^{1}$ and scour data collection; (9) avoidance of factors that might complicate or hinder scour measurement; and (10) safety considerations for data-collection personnel.

An onsite field reconnaissance, including photographic documentation, was made of more than 120 sites in Missouri. Suitable sites were selected as potential bridge-scour study sites. Of these, scour data were collected at 10 of the selected sites and are reported in this report.

Onsite surveys were made at the selected sites to obtain detailed location, cross-section, bridge geometry, and bed-material data. Cross sections were obtained during low flows along the upstream and downstream sides of each bridge during pre- and post-flood visits to establish existing conditions. Reference marks and stationing were established on the bridge handrails for vertical and horizontal control. Bed-material samples were collected to determine the representative size and gradation of streambed material as outlined by Guy and Norman (1970). The bed-material samples were analyzed using methods described by Guy (1969).

Cross sections, along upstream and downstream sides of bridges, were measured during high flows using Fathometers or by manual sounding. These cross sections were plotted to determine the location and depth of the scour holes. The cross-section measurements included streambed elevations at and on either side of the bridge piers. The Fathometers were capable of producing continuous soundings, whereas manual methods resulted in discrete soundings of cross sections. Plots of bridge details and stream cross sections prepared from these data provide comparisons that illustrate the results of scour processes for different hydraulic and geometric conditions.

For this report, the depth of a scour hole was calculated as the difference between the elevation of the projected channel cross section across the scour hole and the lowest measured streambed elevation of the hole (Landers and Mueller, 1993). This projected channel cross section represents the concurrent ambient bed level at the scour hole. Flow depth was calculated as the difference between the elevation of the water surface and the elevation of the projected channel cross section at the scour hole. For historical flood measurements, the maximum depth of a scour hole was assumed to be at the lowest streambed elevation recorded.

${ }^{1}$ The use of trade names in this report is for identification purposes only and does not constitute endorsement by the U.S. Geological Survey. 
Discharge and velocity were determined using standard streamflow-gaging procedures described by Rantz and others (1982). The velocity variable used in existing scour equations is the average velocity of the vertical section immediately upstream from the pier. For scour measurements along the downstream side of the bridge, average velocity at the pier was calculated as the average of the representative velocities in the vertical sections on each side of the pier.

This study relied on the use of portable Fathometers (Gorin and Haeni, 1989), supplemented by manual depth measuring (sounding) equipment, to measure scour at bridge piers. Fathometers were used to measure scour at cross sections on upstream and downstream sides of bridges. More than 130 channel cross sections at bridges were sounded using Fathometers during 1990-92. The Fathometer proved to be an accurate tool for collecting scour data, but its application had limitations under certain onsite conditions (including high velocities and extreme streamflow turbulence). However, the alternative to using Fathometers was to manually sound cross sections at 1 - or 2 -foot horizontal intervals near the piers. The Fathometer proved to be much faster and, under some onsite conditions, produced better results than did manual sounding methods. However, manual soundings were needed to supplement Fathometer soundings and where conditions prevented effective use of the Fathometer.

The portable Fathometer used in this study was an EAGLE MACH I sonic depth sounder, manufactured by Lowrance Electronics, Inc., which is a graphical Fathometer that was relatively inexpensive and available commercially. The Fathometer was temporarily mounted on the side of the stream gaging truck or put in the truck cab. The Fathometer transducer was mounted on the bottom of a standard sounding weight that was suspended by reel and cable from a truck-mounted boom so that it could be towed through the water as the truck was driven across the bridge (Southard, 1989) creating a continuous, graphical chart of the stream bottom recorded by the Fathometer. A transducer with an 8degree cone angle and a 25-foot lead cable was used for bridge-scour measurements. To allow for varying distances from water surface to bridge deck, the Fathometers were factory calibrated for a 75-foot cable extension between Fathometer and transducer lead cable. The transducer must be submerged below the water surface to operate correctly; during this study, a submergence of about 2 feet produced good results.

Computer programming and data base design provided for digitizing and direct computer entry, storage, retrieval, and plotting of the collected data (for example, Fathometer charts, surveys, manual soundings, and bridge plans) for analysis of scour data. A Fortran computer program was written to compute cross-sectional data from digitized Fathometer charts and to adjust these data for horizontal and vertical control before storage in the computer data base. Channel cross sections from Fathometer charts or manual soundings then were retrieved from the computer data base and plotted to uniform scales for measurement and comparison of recorded scour depths at piers.

A Bridge Scour Data Management System (BSDMS) that has been developed to support preparation, compilation, and analysis of bridge scour measurement data (Landers, 1991) also was used in this study. The BSDMS was developed by the USGS National Scour Study, in cooperation with the Federal Highway Administration to facilitate: (1) the development of improved estimators of scour for specific regions or conditions; (2) the description of scour processes; and (3) the decreased risk from scour at bridges. The computational routines contained therein for scour equations were used to compute scour depth estimates presented in this report.

\section{DESCRIPTION OF STUDY SITES}

Of the several suitable sites where cross-section data were collected, local scour around bridge piers was measured at 10 sites located in two of three major physiographic provinces in Missouri (fig.1). Six of these sites are in the Central Lowland Province in the northern part of the state. Four study sites are in the 
southern part of the state in the Ozark Plateaus Province. Also, the North Cobb Creek near Lebanon site that is described later in the report is located in the Ozark Plateaus Province. No sites were located in the Coastal Plains Province. The scour data collected at these 10 sites formed the data base for the statistical analyses of local scour around bridge piers described in this report.

Drainage area, discharge at times of scour measurement, flood peak discharge, and recurrence interval of the flood peak discharge for the 10 study sites for which scour data were collected are given in table 1 . Of these study sites, eight are at streamflow-gaging stations where previous discharge measurements have been made during large floods. Drainage areas for the 10 sites ranged from 175 square miles for the Big River at Irondale to 6,880 square miles for the Grand River near Sumner. For sites where the recurrence intervals ${ }^{2}$ of the flood peak discharges were determined, the intervals ranged from less than 2 years for several sites to more than 50 years for the Gasconade River near Rich Fountain.

\section{MEASURED SCOUR DEPTHS}

To investigate bridge scour at selected sites on Missouri streams, data were collected during floods (fig. 2) large enough to cause local scour at bridge piers. This local scour involves lowering of the streambed below a natural level in the vicinity of the bridge piers. Scour measurements are needed to determine how and to what depth the streambed scours around bridge piers because of factors, such as increased flow velocities, depths, and turbulence near the upstream side of the bridge and the bridge piers. Scour depths estimated from published scour equations are compared in this report with scour depths measured onsite.

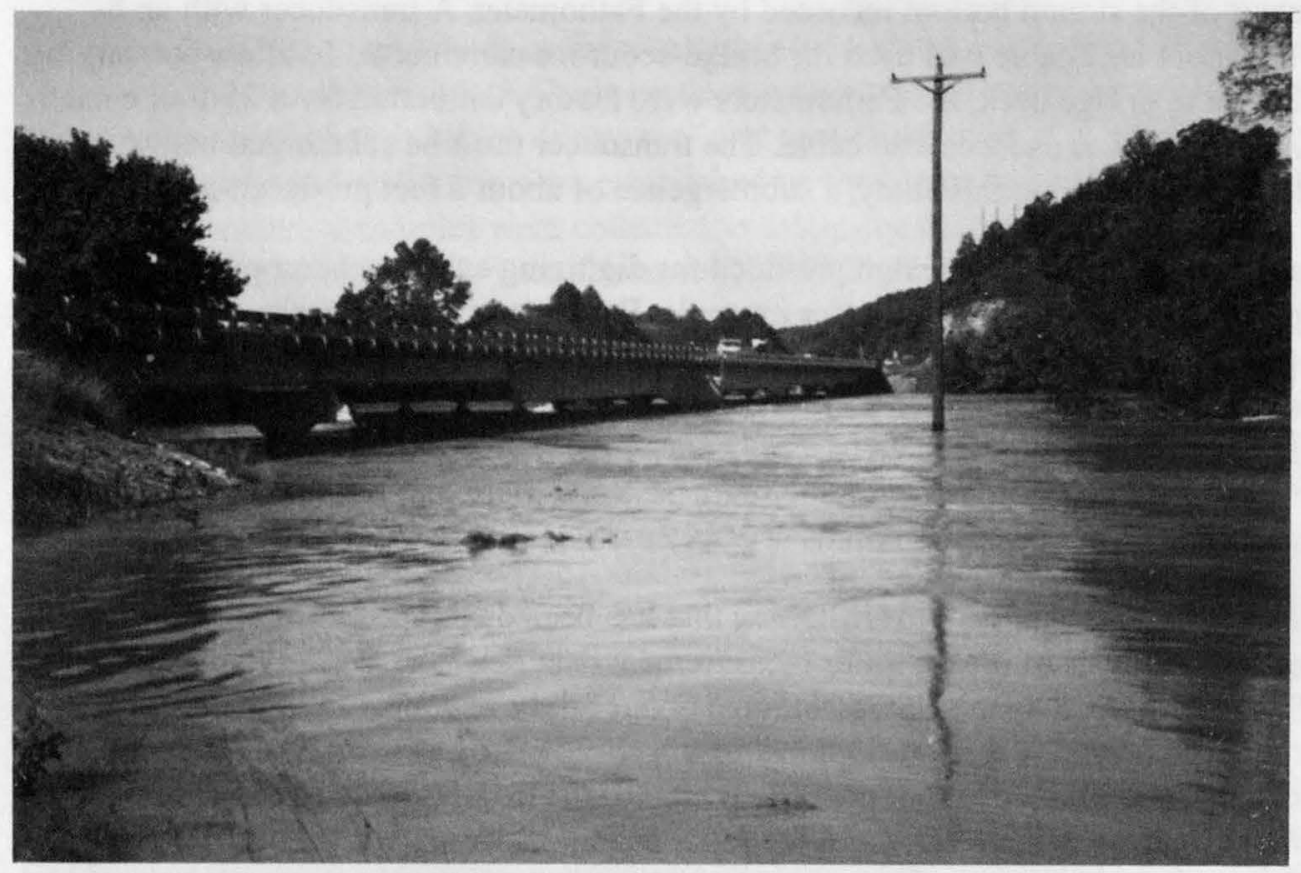

Figure 2. View of downstream side of bridge at time of scour measurement made during the flood of September 28, 1993, at the Gasconade River near Rich Fountain, Missouri (station number 06934000).

\footnotetext{
${ }^{2}$ The recurrence interval, as applied to floods, is the average number of years within which a given flood peak will be equaled or exceeded once. For example, a 100-year flood discharge will be exceeded on the average of once in 100 years. In terms of probability, there is a 1 percent chance that such a flood will occur in any year. A given flood magnitude can be exceeded at any time during a given period.
} 
Table 1.-Summary of discharge data at selected sites in Missouri

[USGS, U.S. Geological Survey; $\mathrm{mi}^{2}$, square miles; $\mathrm{ft}^{3} / \mathrm{s}$, cubic feet per second; <, less than; --, no data; >, greater than]

\begin{tabular}{|c|c|c|c|c|c|c|c|}
\hline $\begin{array}{c}\text { USGS } \\
\text { station } \\
\text { number } \\
\text { (fig. 1) }\end{array}$ & Station name and location & $\begin{array}{c}\text { Drainage } \\
\text { area } \\
\left(\mathrm{mi}^{2}\right)\end{array}$ & $\begin{array}{c}\text { Date of } \\
\text { measurement }\end{array}$ & Time & $\begin{array}{c}\text { Scour } \\
\text { measurement } \\
\text { discharge } \\
\left(\mathrm{ft}^{3} / \mathrm{s}\right)\end{array}$ & $\begin{array}{c}\text { Flood } \\
\text { peak } \\
\text { discharge } \\
\left(\mathrm{ft}^{3} / \mathrm{s}\right)\end{array}$ & $\begin{array}{c}\text { Recurrence } \\
\text { interval } \\
\text { (years) }\end{array}$ \\
\hline \multirow[t]{14}{*}{05502500} & North Fork Salt River near Shelbina (State Highway 15) & 481 & $03-15-90$ & 1145 & 5,510 & -- & -- \\
\hline & & & & 1620 & 5,450 & -- & -- \\
\hline & & & & 1838 & 5,570 & - & -- \\
\hline & & & 03-16-90 & 0045 & -- & 5,750 & $<2$ \\
\hline & & & & 0846 & 5,450 & - & -- \\
\hline & & & & 0950 & 5,510 & -- & -- \\
\hline & & & $11-18-91$ & 1547 & 5,270 & -- & -- \\
\hline & & & $11-19-91$ & -- & -- & 5,750 & $<2$ \\
\hline & & & $11-22-92$ & 0715 & -- & 8,600 & 3.4 \\
\hline & & & $11-23-92$ & 0850 & 6,670 & -- & -- \\
\hline & & & $12-16-92$ & 1011 & 5,870 & -- & -- \\
\hline & & & & 1028 & 5,870 & -- & -- \\
\hline & & & & 1045 & 5,870 & - & -- \\
\hline & & & $12-17-92$ & 0215 & - & 6,790 & 2.3 \\
\hline \multirow[t]{2}{*}{05508000} & Salt River near New London (U.S. Highway 61) & 2,480 & $07-24-91$ & 1800 & -- & 7,800 & $<2$ \\
\hline & & & $07-25-91$ & 1123 & 6,600 & - & - \\
\hline \multirow[t]{4}{*}{06817600} & Nodaway River near Skidmore (Highway DD) & 1,350 & $11-20-92$ & 1123 & 8,000 & -- & -- \\
\hline & & & & 1357 & 8,000 & -- & -- \\
\hline & & & $12-14-92$ & 1154 & -- & -- & -- \\
\hline & & & & 1209 & -- & - & -- \\
\hline \multirow[t]{4}{*}{06902000} & Grand River near Sumner (State Highway 139) & 6,880 & 03-16-90 & - & -- & 50,100 & $<2$ \\
\hline & & & & 1400 & $\mathrm{a}_{34,000}$ & - & - \\
\hline & & & & -- & - & 76,600 & 4 \\
\hline & & & 05-07-91 & 1452 & a39,600 & - & -- \\
\hline
\end{tabular}


Table 1.--Summary of discharge data at selected sites in Missouri-Continued

\begin{tabular}{|c|c|c|c|c|c|c|c|}
\hline $\begin{array}{l}\text { USGS } \\
\text { station } \\
\text { number } \\
\text { (fig. 1) }\end{array}$ & Station name and location & $\begin{array}{c}\text { Drainage } \\
\text { area } \\
\left(\mathrm{mi}^{2}\right)\end{array}$ & $\begin{array}{l}\text { Date of } \\
\text { measurement }\end{array}$ & Time & $\begin{array}{c}\text { Scour } \\
\text { measurement } \\
\text { discharge } \\
\left(\mathrm{ft}^{3} / \mathrm{s}\right)\end{array}$ & $\begin{array}{c}\text { Flood } \\
\text { peak } \\
\text { discharge } \\
\left(\mathrm{ft}^{3} / \mathrm{s}\right)\end{array}$ & $\begin{array}{l}\text { Recurrence } \\
\text { interval } \\
\text { (years) }\end{array}$ \\
\hline \multirow[t]{2}{*}{06902000} & Grand River near Sumner (State Highway 139)--Continued & 6,880 & $04-23-92$ & -- & -- & 72,500 & 4 \\
\hline & & & & 1305 & -- & -- & -- \\
\hline \multirow[t]{6}{*}{06904500} & Chariton River at Novinger (State Highway 6) & 1,370 & $04-12-92$ & 1330 & 7,030 & -- & $\mathrm{b}$ \\
\hline & & & 04-13-92 & 1335 & 5,140 & -- & -- \\
\hline & & & $12-16-92$ & 0330 & -- & 12,600 & $\mathrm{~b}$ \\
\hline & & & & 1322 & 7,170 & -- & -- \\
\hline & & & $07-13-93$ & 1445 & 17,400 & -- & -- \\
\hline & & & & 0030 & -- & 20,900 & $\mathrm{~b}$ \\
\hline \multirow{3}{*}{06905500} & Chariton River near Prairie Hill (State Highway 129) & 1,870 & $07-07-93$ & 2034 & 23,100 & -- & -- \\
\hline & & & & 0915 & 27,200 & -- & -- \\
\hline & & & $07-13-93$ & 1945 & -- & 31,300 & $\mathrm{~b}$ \\
\hline \multirow[t]{2}{*}{06932000} & Little Piney Creek at Newburg (Highway P-T) & 200 & 05-03-90 & -- & -- & 5,480 & $<2$ \\
\hline & & & & 1105 & 5,360 & -- & -- \\
\hline \multirow{7}{*}{, } & & & & 1132 & 5,160 & -- & -- \\
\hline & & & 05-13-91 & 1503 & 9,290 & -- & -- \\
\hline & & & & 1517 & 8,830 & -- & -- \\
\hline & & & & 1830 & -- & 21,300 & 15 \\
\hline & & & & 1930 & 15,700 & -- & -- \\
\hline & & & & 1949 & 15,400 & -- & -- \\
\hline & & & $05-14-91$ & 0006 & 9,050 & -- & - \\
\hline \multirow[t]{4}{*}{06933850} & Gasconade River near Vienna (State Highway 42) & 3,050 & $01-07-93$ & - & -- & ${ }^{c} 40,000$ & 3.4 \\
\hline & & & & 1045 & 30,800 & -- & -- \\
\hline & & & $11-24-92$ & 0955 & 26,400 & -- & -- \\
\hline & & & $11-24-92$ & -- & -- & $c_{31,500}$ & 2.3 \\
\hline
\end{tabular}


Table 1.-Summary of discharge data at selected sites in Missouri-Continued

\begin{tabular}{|c|c|c|c|c|c|c|c|}
\hline $\begin{array}{c}\text { USGS } \\
\text { station } \\
\text { number } \\
\text { (fig. 1) }\end{array}$ & Station name and location & $\begin{array}{c}\text { Drainage } \\
\text { area } \\
\left(\mathrm{mi}^{2}\right)\end{array}$ & $\begin{array}{c}\text { Date of } \\
\text { measurement }\end{array}$ & Time & $\begin{array}{c}\text { Scour } \\
\text { measurement } \\
\text { discharge } \\
\left(\mathrm{ft}^{3} / \mathrm{s}\right)\end{array}$ & $\begin{array}{c}\text { Flood } \\
\text { peak } \\
\text { discharge } \\
\left(\mathrm{ft}^{3} / \mathrm{s}\right)\end{array}$ & $\begin{array}{c}\text { Recurrence } \\
\text { interval } \\
\text { (years) }\end{array}$ \\
\hline \multirow[t]{3}{*}{06934000} & Gasconade River near Rich Fountain (State Highway 89) & 3,180 & $01-07-93$ & 1256 & 40,200 & 40,200 & 3.4 \\
\hline & & & $09-28-93$ & 1328 & 101,000 & -- & -- \\
\hline & & & & 1000 & -- & 106,000 & 54 \\
\hline \multirow[t]{6}{*}{07017200} & Big River at Irondale (Highway U) & 175 & $03-18-87$ & 1120 & 2,030 & -- & -- \\
\hline & & & $05-03-90$ & 1130 & -- & 8,720 & $<2$ \\
\hline & & & & 1304 & 6,480 & -- & - \\
\hline & & & $11-01-91$ & -- & -- & 1,780 & $<2$ \\
\hline & & & & 1106 & 1,540 & -- & -- \\
\hline & & & & 1220 & 1,330 & -- & -- \\
\hline
\end{tabular}

a Overflow channel not included.

${ }^{\mathrm{b}}$ Regulated, not determined.

${ }^{c}$ Estimated. 
Scour measurements made during this study and review of historical discharge measurements at streamflow-gaging stations resulted in 54 sets of data collected during 24 floods (table 2). Measured scour holes at piers ranged from 0.5 to 7.1 feet deep. The deepest scour hole ( 7.1 feet) was determined from Fathometer soundings along the upstream side of the bridge at the Grand River near Sumner during the flood on March 16, 1990. Local scour depths presented in this report (table 2) are scour depths attributable to local scour only, at the time of and under conditions existing during the scour measurement. Flow disturbances at and near piers that cause local scour to occur are shown in figures 3 and 4 .

Total scour, particularly in sand channels, can be the result of either general, contraction, or local scour, or a combination thereof. Although relatively small local scour depths may be evident, total depths of scour that included general or contraction scour may have been much greater, as evidenced by a general lowering of the streambed. The effects of local scour caused by the bridge piers can be measured only after being superimposed on this lowered streambed. Conversely, in some cases local scour measurements may have been made during times when the streambed at the bridge may have been in a temporary state of aggradation (raising).

Of the 10 sites for which local scour is reported, 4 sites are on streams that might be categorized as sand channels. As a consequence, some measurements were made during conditions of changing sandchannel dune regimes (Lagasse and others, 1991, p. 37-40), making the proper separation of local scour from other scour mechanisms difficult. Sand-bed channels add a degree of complexity to scour-depth measurement that only becomes apparent on plotting successive cross sections at the site under varying flow conditions.

Woody debris accumulation at bridges has caused maintenance problems, scour, and bridge failure. Large woody debris is the dominant type of debris involved. The USGS, in cooperation with the Federal Highway Administration, is investigating the causes and effects of debris accumulation at bridges (T.H. Diehl, U.S. Geological Survey, written commun., 1992).

Debris flow and accumulations hindered or prevented scour data collection at several sites during this study. Flowing and accumulated debris create problems with making velocity and depth measurements around piers. Extreme examples of debris as a hinderance to data collection are indicated by photographs of flowing woody debris on the Grand River near Sumner (fig. 5), debris accumulation on the piers during flooding on the Chariton River near Prairie Hill (figs. 6 and 7), and debris accumulation after a flood on the Chariton River at Novinger (fig. 8). Such debris flows and accumulations on bridge piers often had such an effect on the measured scour that using these data for comparison with published scour equations was not feasible.

One effect of lodged debris, with respect to scour, is that it effectively increases pier width and decreases the bridge-opening area. At some bridges located on sand channel streams in northern Missouri, debris accumulated on the piers during larger floods to such an extent that the bridge opening was contracted to a degree that caused or increased scour remote from the piers. When such partial blockage of the bridge opening occurs, increased contraction scour can occur, resulting in scour depths that are much greater than would be attributable only to local scour.

This phenomenon of increased scour has been shown to occur during extreme floods at the Chariton River near Prairie Hill site by comparison of cross-sectional data from discharge measurement soundings made before, during, and after flood peaks. For example, during the July 8, 1993, flood, the streambed was lowered, as shown in figure 9 , by about 19 feet at the downstream side of the bridge in comparison to streambed levels recorded on June 9, 1993. This temporary degradation is attributable to combined effects of contracted flow at the bridge and partial blockage of the bridge opening by woody debris (figs. 6 and 7). A cross section obtained during a discharge measurement on August 19, 1993, indicated (fig. 9) that the 
Table 2.-Summary of pier, scour, and hydraulic data collected at selected sites in Missouri

[USGS, U.S. Geological Survey; ft, feet; ft/s, feet per second; US, upstream; DS, downstream; data are ordered by pier identification number]

\begin{tabular}{|c|c|c|c|c|c|c|c|c|c|c|c|}
\hline \multirow[b]{2}{*}{$\begin{array}{l}\text { USGS } \\
\text { station } \\
\text { number } \\
\text { (fig. 1) }\end{array}$} & \multirow[b]{2}{*}{$\begin{array}{c}\text { Date of } \\
\text { measurement }\end{array}$} & \multicolumn{4}{|c|}{ Pier data } & \multirow[b]{2}{*}{$\begin{array}{c}\text { Relation of } \\
\text { measurement } \\
\text { to } \\
\text { bridge }\end{array}$} & \multirow[b]{2}{*}{$\begin{array}{l}\text { Measured } \\
\text { scour } \\
\text { depth } \\
\text { (ft) }\end{array}$} & \multirow[b]{2}{*}{$\begin{array}{l}\text { Median bed- } \\
\text { material } \\
\text { diameter } \\
\text { (ft) }\end{array}$} & \multicolumn{3}{|c|}{ Hydraulic data at scour hole section } \\
\hline & & Time & $\begin{array}{l}\text { Identification } \\
\text { number }\end{array}$ & $\begin{array}{l}\text { Type of } \\
\text { nose }\end{array}$ & $\begin{array}{l}\text { Width } \\
\text { normal to } \\
\text { flow } \\
\text { (ft) }\end{array}$ & & & & $\begin{array}{l}\text { Flow } \\
\text { depth } \\
\text { (ft) }\end{array}$ & $\begin{array}{c}\text { Average } \\
\text { velocity } \\
\text { (ft/s) }\end{array}$ & $\begin{array}{c}\text { Flow angle } \\
\text { (degrees) }\end{array}$ \\
\hline \multicolumn{12}{|c|}{ North Fork Salt River near Shelbina } \\
\hline \multirow[t]{17}{*}{05502500} & $03-15-90$ & 1838 & 1 & Round & 3.0 & US & 0.7 & 0.00157 & 12.1 & 2.9 & 0 \\
\hline & $03-16-90$ & 0846 & 1 & Round & 3.0 & US & 6 & .00157 & 11.4 & 3.0 & 0 \\
\hline & & 0950 & 1 & Round & 3.0 & DS & 1.0 & .00157 & 11.2 & 3.0 & 0 \\
\hline & $11-18-91$ & 1547 & 1 & Round & 3.0 & US & .9 & .00157 & 11.8 & 3.0 & 0 \\
\hline & $11-23-92$ & 0850 & 1 & Round & 3.0 & US & .5 & .00157 & 13.5 & 3.1 & 0 \\
\hline & $12-16-92$ & 1028 & 1 & Round & 3.0 & US & 1.4 & .00157 & 12.7 & 3.0 & 0 \\
\hline & & 1045 & 1 & Round & 3.0 & US & .9 & .00157 & 13.0 & 3.0 & 0 \\
\hline & $03-15-90$ & 1620 & 2 & Round & 3.0 & US & .8 & .00157 & 11.6 & 3.3 & 0 \\
\hline & & 1645 & 2 & Round & 3.0 & DS & .8 & .00157 & 11.2 & 3.5 & 0 \\
\hline & & 1838 & 2 & Round & 3.0 & US & 1.1 & .00157 & 11.8 & 3.7 & 0 \\
\hline & $03-16-90$ & 0846 & 2 & Round & 3.0 & US & .8 & .00157 & 11.6 & 3.3 & 0 \\
\hline & & 0950 & 2 & Round & 3.0 & DS & 1.2 & .00157 & 10.8 & 3.2 & 0 \\
\hline & $11-18-91$ & 1547 & 2 & Round & 3.0 & US & .8 & .00157 & 12.2 & 3.2 & 0 \\
\hline & $11-23-92$ & 0850 & 2 & Round & 3.0 & US & .9 & .00157 & 14.1 & 3.7 & 0 \\
\hline & $12-16-92$ & 1011 & 2 & Round & 3.0 & DS & .9 & .00157 & 12.0 & 3.5 & 0 \\
\hline & & 1028 & 2 & Round & 3.0 & US & 1.0 & .00157 & 13.1 & 3.5 & 0 \\
\hline & & 1045 & 2 & Round & 3.0 & US & .7 & .00157 & 13.3 & 3.5 & 0 \\
\hline \multicolumn{12}{|c|}{ Salt River near New London } \\
\hline 05508000 & $07-25-91$ & 1123 & 3 & Round & 3.5 & US & 1.3 & .03675 & 7.9 & 3.2 & 0 \\
\hline \multicolumn{12}{|c|}{ Nodaway River near Skidmore } \\
\hline \multirow[t]{6}{*}{06817600} & $11-20-92$ & 1335 & 1 & Round & 3.0 & DS & 1.7 & .00110 & 9.2 & 4.3 & 0 \\
\hline & & 1357 & 1 & Round & 3.0 & US & 2.1 & .00110 & 9.2 & 4.3 & 0 \\
\hline & $12-14-92$ & 1154 & 1 & Round & 3.0 & US & 2.5 & .00110 & 10.6 & 4.2 & 0 \\
\hline & $11-20-92$ & 1335 & 2 & Round & 3.0 & DS & 2.1 & .00110 & 15.1 & 2.8 & 0 \\
\hline & & 1357 & 2 & Round & 3.0 & US & 1.8 & .00110 & 14.9 & 2.8 & 0 \\
\hline & $12-14-92$ & 1209 & 2 & Round & 3.0 & US & 3.3 & .00110 & 13.2 & 4.9 & 0 \\
\hline
\end{tabular}


Table 2.-Summary of pier, scour, and hydraulic data collected at selected sites in Missouri-Continued

\begin{tabular}{|c|c|c|c|c|c|c|c|c|c|c|c|}
\hline \multirow[b]{2}{*}{$\begin{array}{l}\text { USGS } \\
\text { station } \\
\text { number } \\
\text { (fig. 1) }\end{array}$} & \multirow[b]{2}{*}{$\begin{array}{c}\text { Date of } \\
\text { measurement }\end{array}$} & \multicolumn{4}{|c|}{ Pier data } & \multirow[b]{2}{*}{$\begin{array}{c}\text { Relation of } \\
\text { measurement } \\
\text { to } \\
\text { bridge }\end{array}$} & \multirow[b]{2}{*}{$\begin{array}{l}\text { Measured } \\
\text { scour } \\
\text { depth } \\
\text { (ft) }\end{array}$} & \multirow[b]{2}{*}{$\begin{array}{c}\text { Median bed- } \\
\text { material } \\
\text { diameter } \\
(\mathrm{ft})\end{array}$} & \multicolumn{3}{|c|}{ Hydraulic data at scour hole section } \\
\hline & & Time & $\begin{array}{c}\text { Identification } \\
\text { number }\end{array}$ & $\begin{array}{l}\text { Type of } \\
\text { nose }\end{array}$ & $\begin{array}{l}\text { Width } \\
\text { normal to } \\
\text { flow } \\
\text { (ft) }\end{array}$ & & & & $\begin{array}{c}\text { Flow } \\
\text { depth } \\
\text { (ft) }\end{array}$ & $\begin{array}{c}\text { Average } \\
\text { velocity } \\
\text { (ft/s) }\end{array}$ & $\begin{array}{l}\text { Flow angle } \\
\text { (degrees) }\end{array}$ \\
\hline \multicolumn{12}{|c|}{ Grand River near Sumner } \\
\hline \multirow[t]{3}{*}{06902000} & $03-16-90$ & 1400 & 2 & Round & 4.8 & US & 7.1 & 0.00032 & 27.8 & 4.8 & 0 \\
\hline & $05-07-91$ & 1452 & 2 & Round & 4.7 & US & 6.6 & .00032 & 31.6 & 4.4 & 0 \\
\hline & $04-23-92$ & 1305 & 2 & Round & 4.7 & US & 6.5 & .00032 & 30.8 & 4.2 & 0 \\
\hline \multicolumn{12}{|c|}{ Chariton River at Novinger } \\
\hline \multirow[t]{3}{*}{06904500} & 04-13-92 & 1335 & 2 & Round & 4.8 & US & 1.9 & .00095 & 7.0 & 3.4 & 0 \\
\hline & $12-16-92$ & 1322 & 2 & Round & 4.6 & US & 3.4 & .00095 & 7.8 & 2.7 & 0 \\
\hline & $07-13-93$ & 1445 & 2 & Round & 4.2 & US & 1.9 & .00095 & 17.6 & 3.9 & 0 \\
\hline \multicolumn{12}{|c|}{ Chariton River near Prairie Hill } \\
\hline 06905500 & $07-07-93$ & 2034 & 3 & Round & 3.9 & US & 3.2 & .00095 & 22.7 & 4.8 & 0 \\
\hline y & $07-08-93$ & 0915 & 3 & Round & 4.1 & DS & 4.7 & .00095 & 31.3 & 4.9 & 0 \\
\hline \multicolumn{12}{|c|}{ Little Piney Creek at Newburg } \\
\hline \multirow[t]{12}{*}{06932000} & $05-03-90$ & 1105 & 1 & Round & 3.6 & DS & .7 & .00174 & 8.8 & 5.7 & 0 \\
\hline & & 1132 & 1 & Round & 3.6 & US & 1.1 & .00174 & 10.3 & 5.7 & 0 \\
\hline & $05-13-91$ & 1503 & 1 & Round & 3.5 & DS & 1.5 & .00174 & 12.2 & 7.1 & 0 \\
\hline & & 1517 & 1 & Round & 3.5 & US & 2.4 & .00174 & 13.6 & 7.0 & 0 \\
\hline & & 1930 & 1 & Round & 3.4 & DS & 2.4 & .00174 & 14.2 & 7.5 & 0 \\
\hline & & 1949 & 1 & Round & 3.4 & US & 1.8 & .00174 & 15.8 & 7.6 & 0 \\
\hline & 05-14-91 & 0006 & 1 & Round & 3.6 & US & 1.4 & .00174 & 13.6 & 7.0 & 0 \\
\hline & $05-13-91$ & 1503 & 2 & Round & 3.5 & DS & .8 & .00174 & 11.8 & 3.4 & 0 \\
\hline & & 1517 & 2 & Round & 3.4 & US & 1.7 & .00174 & 11.0 & 3.1 & 0 \\
\hline & & 1930 & 2 & Round & 3.4 & DS & .7 & .00174 & 14.1 & 5.4 & 0 \\
\hline & & 1949 & 2 & Round & 3.4 & US & 1.0 & .00174 & 13.7 & 5.3 & 0 \\
\hline & $05-14-91$ & 0006 & 2 & Round & 3.5 & US & .9 & .00174 & 11.9 & 3.4 & 0 \\
\hline \multicolumn{12}{|c|}{ Gasconade River near Vienna } \\
\hline \multirow[t]{3}{*}{06933850} & $11-24-92$ & 0955 & 4 & Round & 3.5 & US & .7 & .04265 & 15.0 & 2.9 & 0 \\
\hline & $01-07-93$ & 1023 & 4 & Round & 3.4 & US & 1.0 & .04265 & 17.0 & 2.8 & 0 \\
\hline & & 1103 & 4 & Round & 3.4 & DS & 1.9 & .04265 & 17.0 & 2.8 & 0 \\
\hline
\end{tabular}


Table 2.--Summary of pier, scour, and hydraulic data collected at selected sites in Missouri-Continued

\begin{tabular}{|c|c|c|c|c|c|c|c|c|c|c|c|}
\hline \multirow[b]{2}{*}{$\begin{array}{l}\text { USGS } \\
\text { station } \\
\text { number } \\
\text { (fig. 1) }\end{array}$} & \multirow[b]{2}{*}{$\begin{array}{c}\text { Date of } \\
\text { measurement }\end{array}$} & \multicolumn{4}{|c|}{ Pier data } & \multirow[b]{2}{*}{$\begin{array}{c}\text { Relation of } \\
\text { measurement } \\
\text { to } \\
\text { bridge }\end{array}$} & \multirow[b]{2}{*}{$\begin{array}{l}\text { Measured } \\
\text { scour } \\
\text { depth } \\
\text { (ft) }\end{array}$} & \multirow[b]{2}{*}{$\begin{array}{l}\text { Median bed- } \\
\text { material } \\
\text { diameter } \\
\text { (ft) }\end{array}$} & \multicolumn{3}{|c|}{ Hydraulic data at scour hole section } \\
\hline & & Time & $\begin{array}{l}\text { Identification } \\
\text { number }\end{array}$ & $\begin{array}{l}\text { Type of } \\
\text { nose }\end{array}$ & $\begin{array}{l}\text { Width } \\
\text { normal to } \\
\text { flow } \\
\text { (ft) }\end{array}$ & & & & $\begin{array}{l}\text { Flow } \\
\text { depth } \\
\text { (ft) }\end{array}$ & $\begin{array}{c}\text { Average } \\
\text { velocity } \\
\text { (ft/s) }\end{array}$ & $\begin{array}{c}\text { Flow angle } \\
\text { (degrees) }\end{array}$ \\
\hline \multicolumn{12}{|c|}{ Gasconade River near Rich Fountain } \\
\hline \multirow[t]{3}{*}{06934000} & $09-28-93$ & 1328 & 3 & Round & 4.5 & US & 3.5 & 0.02854 & 32.4 & 4.5 & 0 \\
\hline & $01-07-93$ & 1256 & 4 & Round & 5.0 & US & 2.8 & .02854 & 19.4 & 6.7 & 0 \\
\hline & $09-28-93$ & 1328 & 4 & Round & 4.5 & US & 4.1 & .02854 & 31.0 & 8.5 & 0 \\
\hline \multicolumn{12}{|c|}{ Big River at Irondale } \\
\hline \multirow[t]{4}{*}{07017200} & $03-18-87$ & 1120 & 2 & Round & 5.4 & US & 1.2 & .01804 & 4.1 & 3.5 & 0 \\
\hline & $05-03-90$ & 1304 & 2 & Round & 5.2 & US & 1.3 & .01804 & 8.4 & 6.0 & 0 \\
\hline & $11-01-91$ & 1106 & 2 & Round & 5.4 & US & 1.7 & .01804 & 4.3 & 3.5 & 0 \\
\hline & & 1220 & 2 & Round & 5.4 & US & 1.7 & .01804 & 3.8 & 3.0 & 0 \\
\hline
\end{tabular}




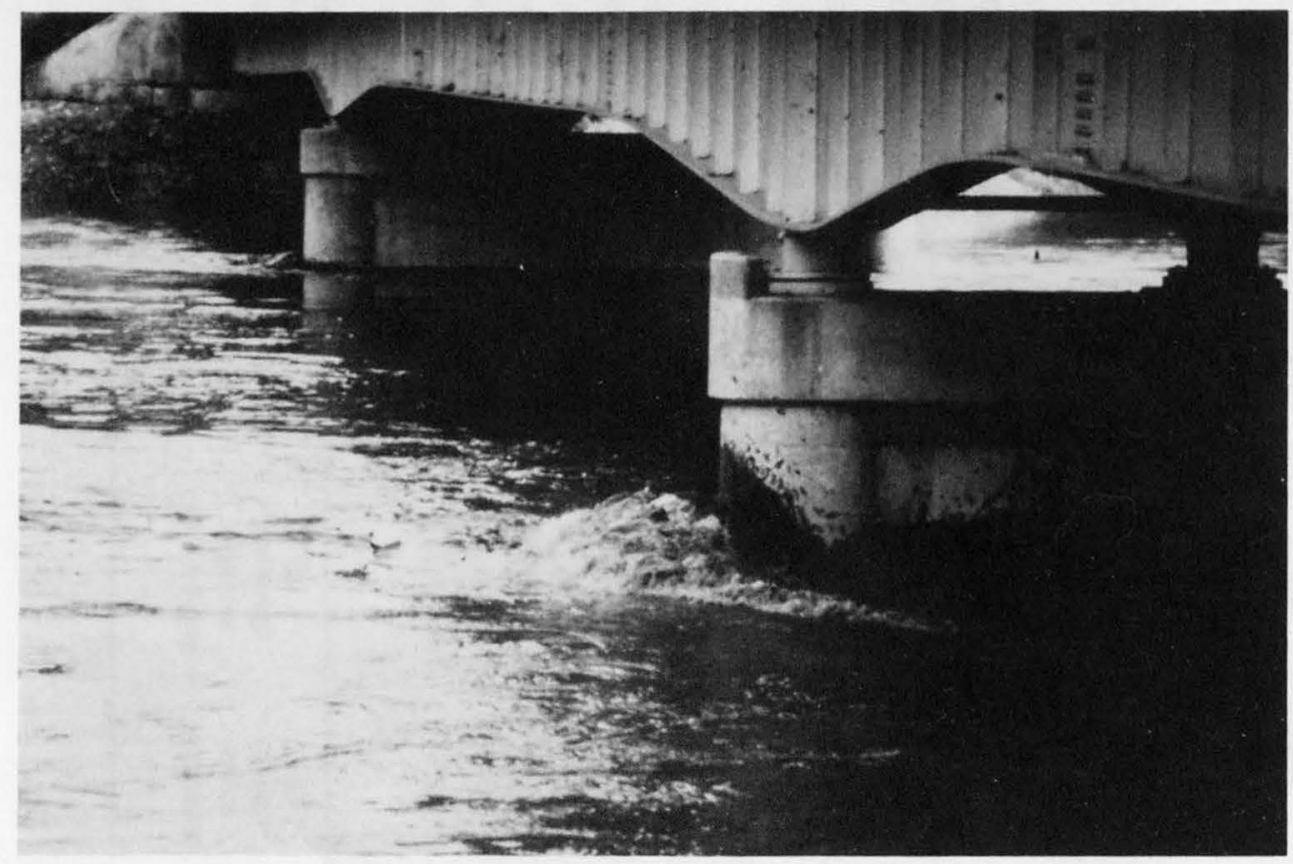

Figure 3. Pileup of water surface at upstream side of bridge pier during the flood of May 13 , 1991, at Little Piney Creek at Newburg, Missouri (station number 06932000).

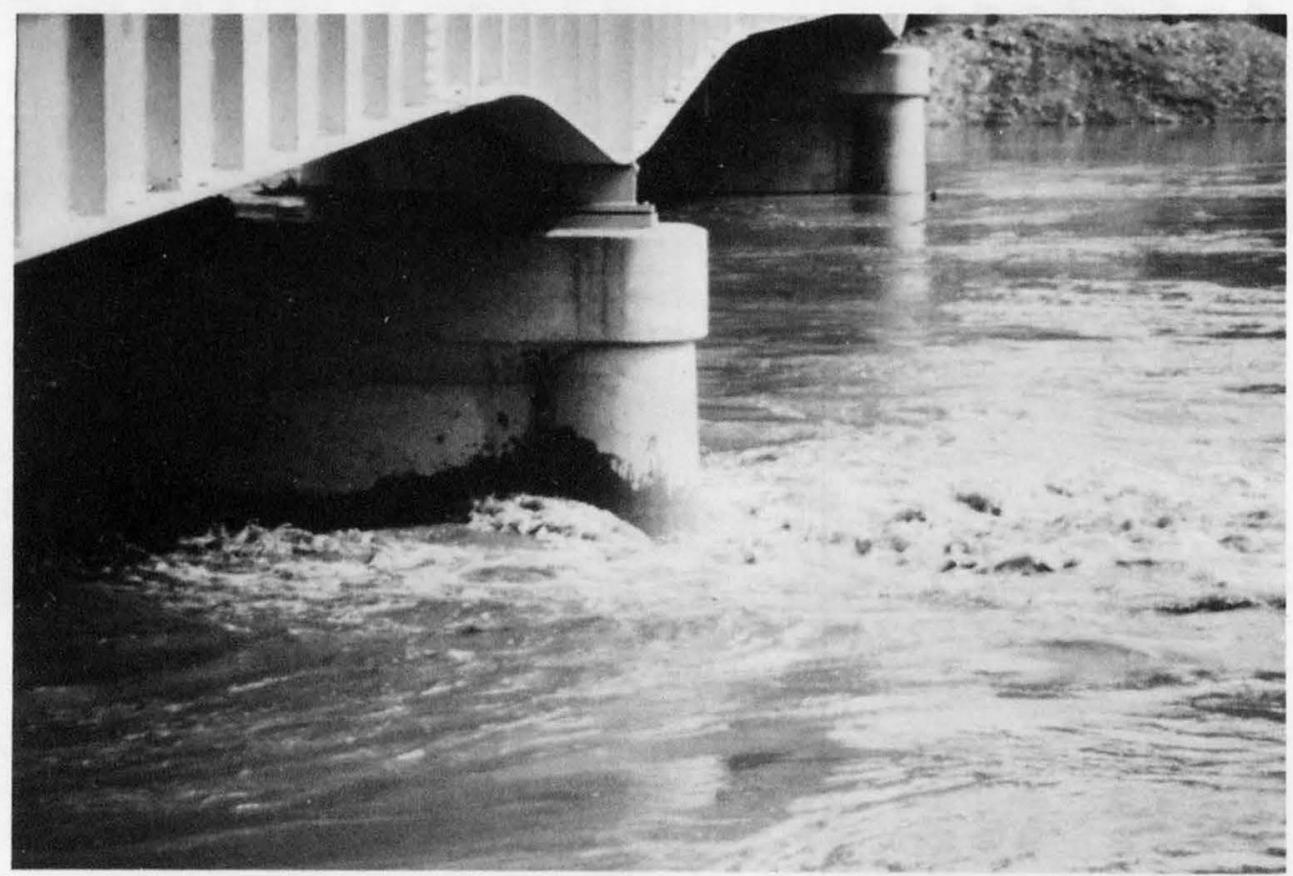

Figure 4. Turbulent flow at and downstream from bridge pier during the flood of May 13,1991, at Little Piney Creek at Newburg, Missouri (station number 06932000). 


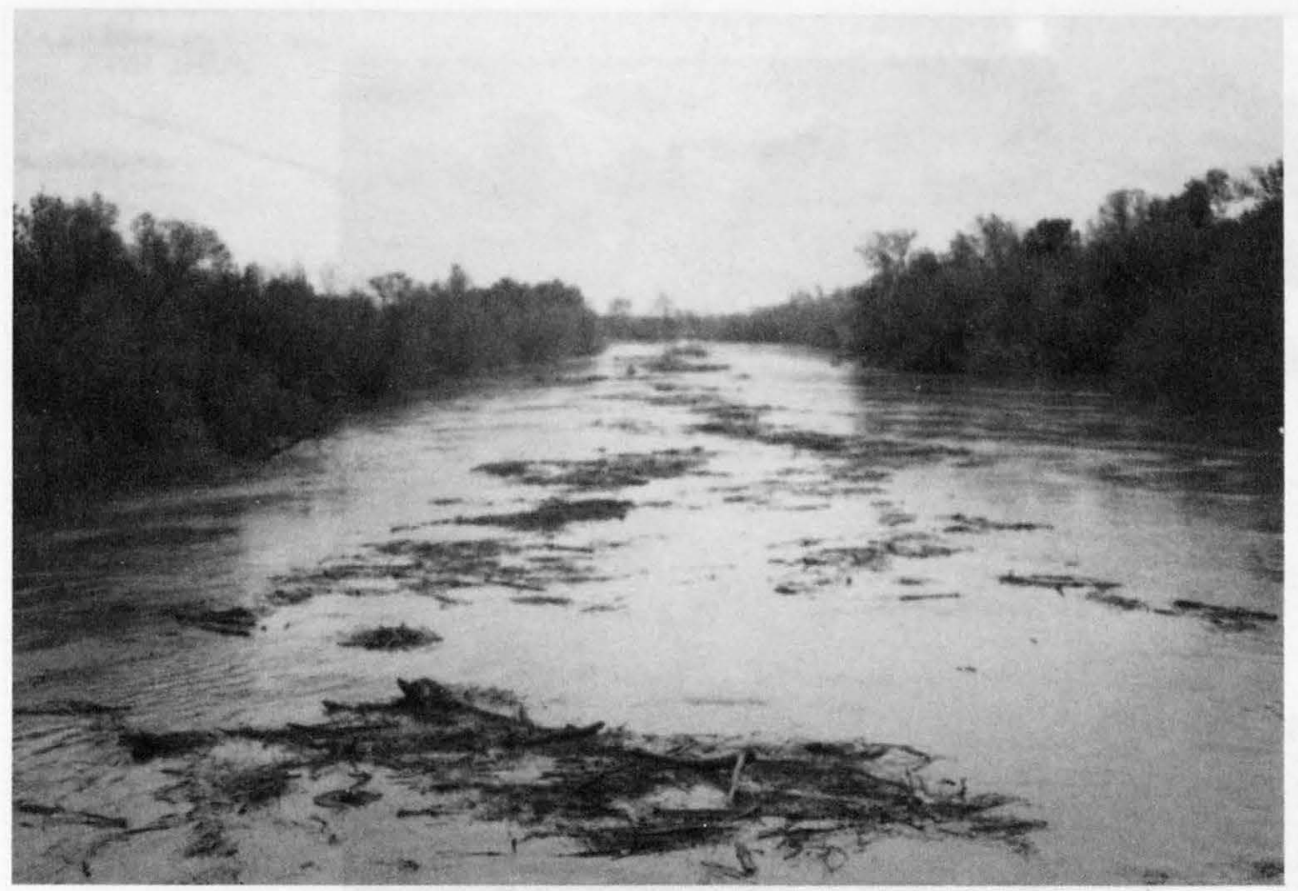

Figure 5. Floating woody debris during the flood crest on April 21, 1991, at the Grand River near Sumner, Missouri (station number 06902000; view downstream from bridge on State Highway 139).

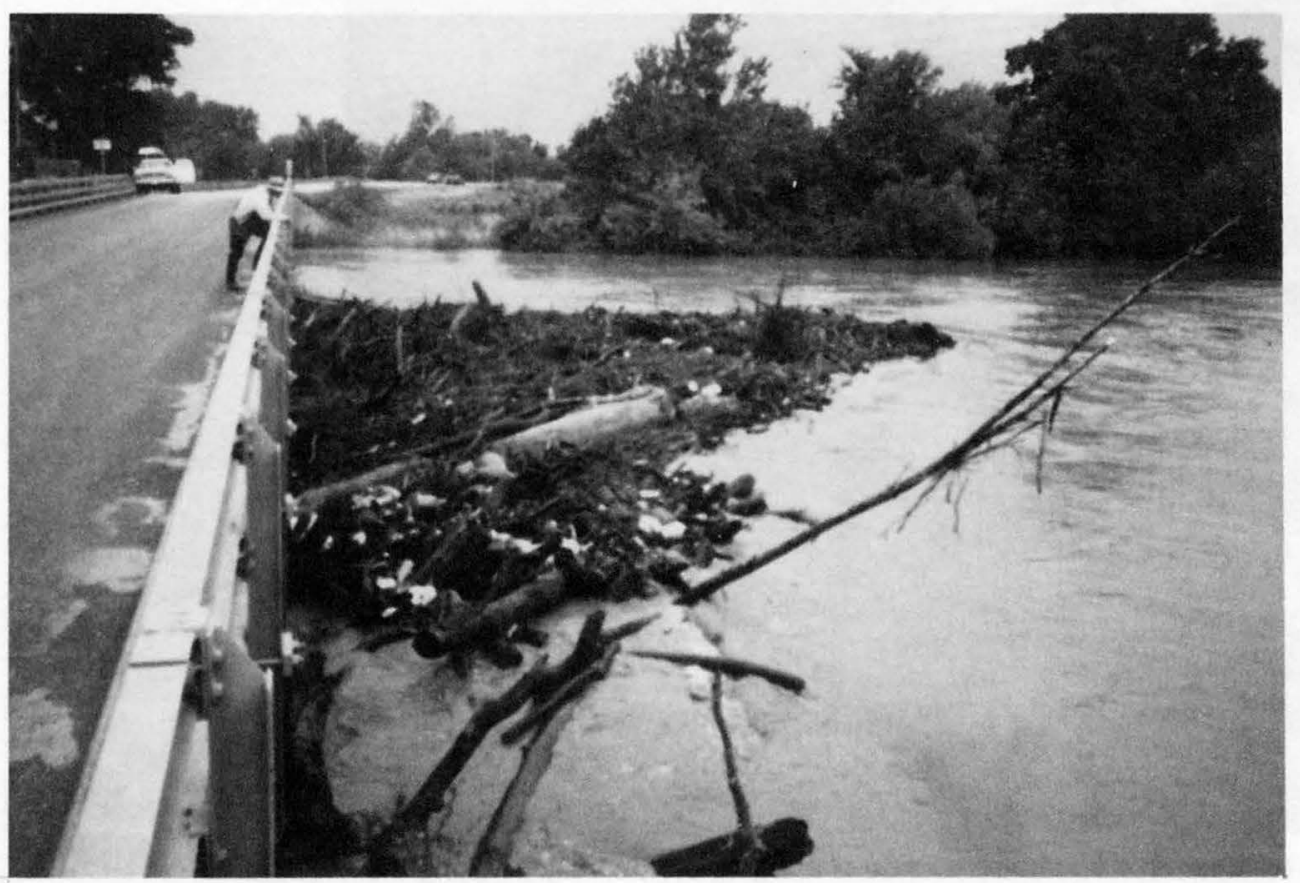

Figure 6. Woody debris accumulated against the center bridge pier partially blocking the bridge opening on the upstream side during the flood of July 8, 1993, at the Chariton River near Prairie Hill, Missouri (station number 06905500). 


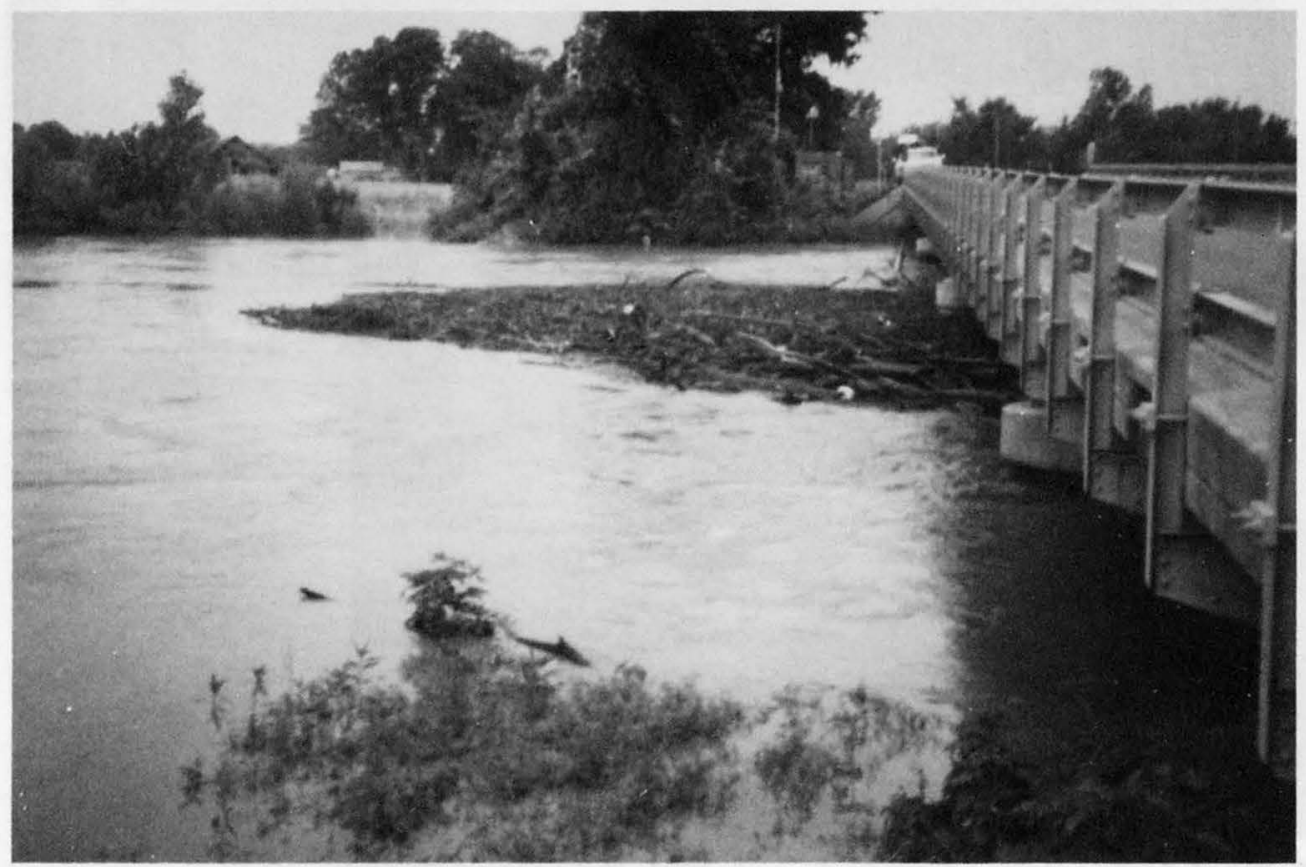

Figure 7. View of the downstream side of bridge and woody debris accumulated on the center pier extending completely through the bridge opening during the flood of July 8, 1993, at the Chariton River near Prairie Hill, Missouri (station number 06905500).

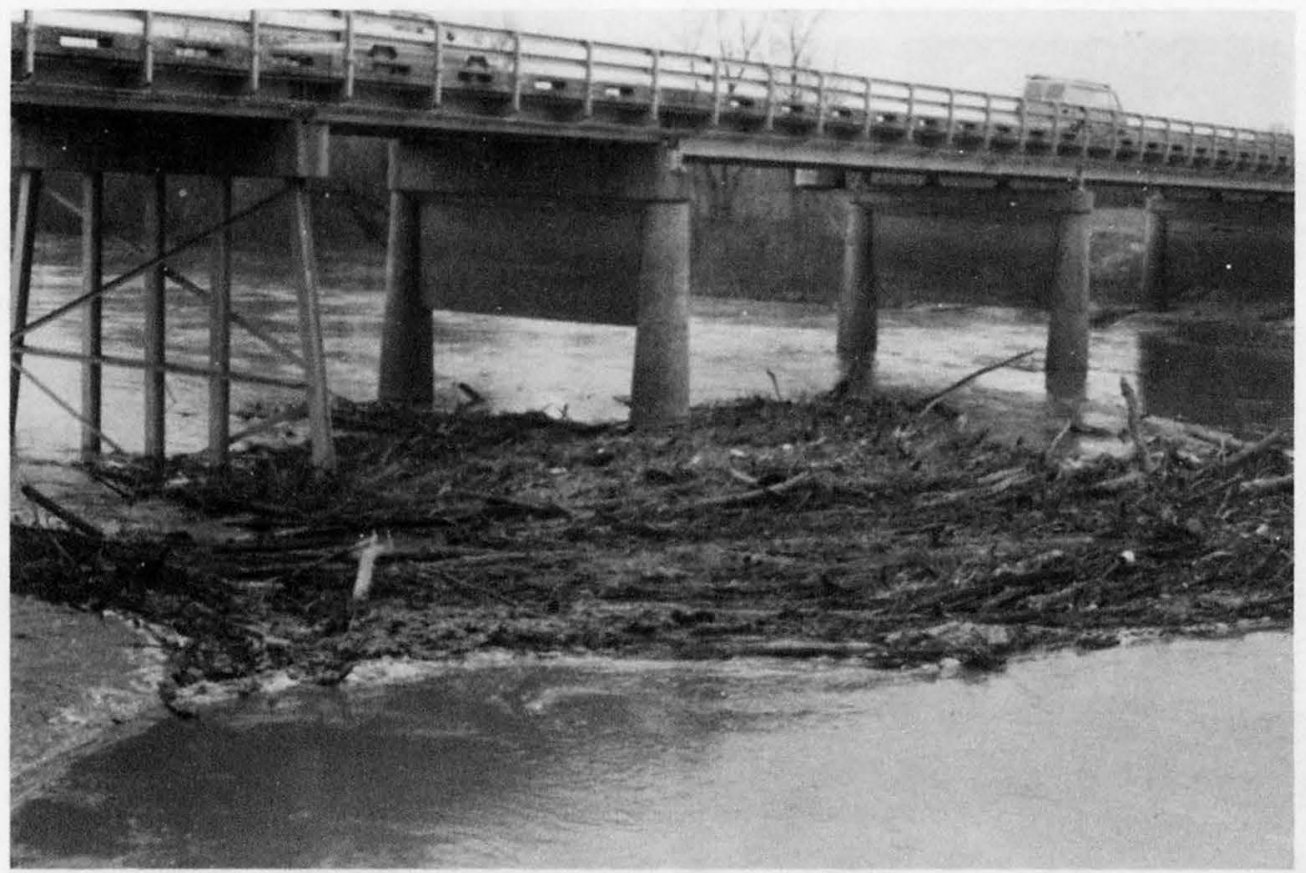

Figure 8. Woody debris accumulated on upstream side of bridge piers partially blocking the bridge opening following the flood of April 12, 1992, at the Chariton River at Novinger, Missouri (station number 06904500). 


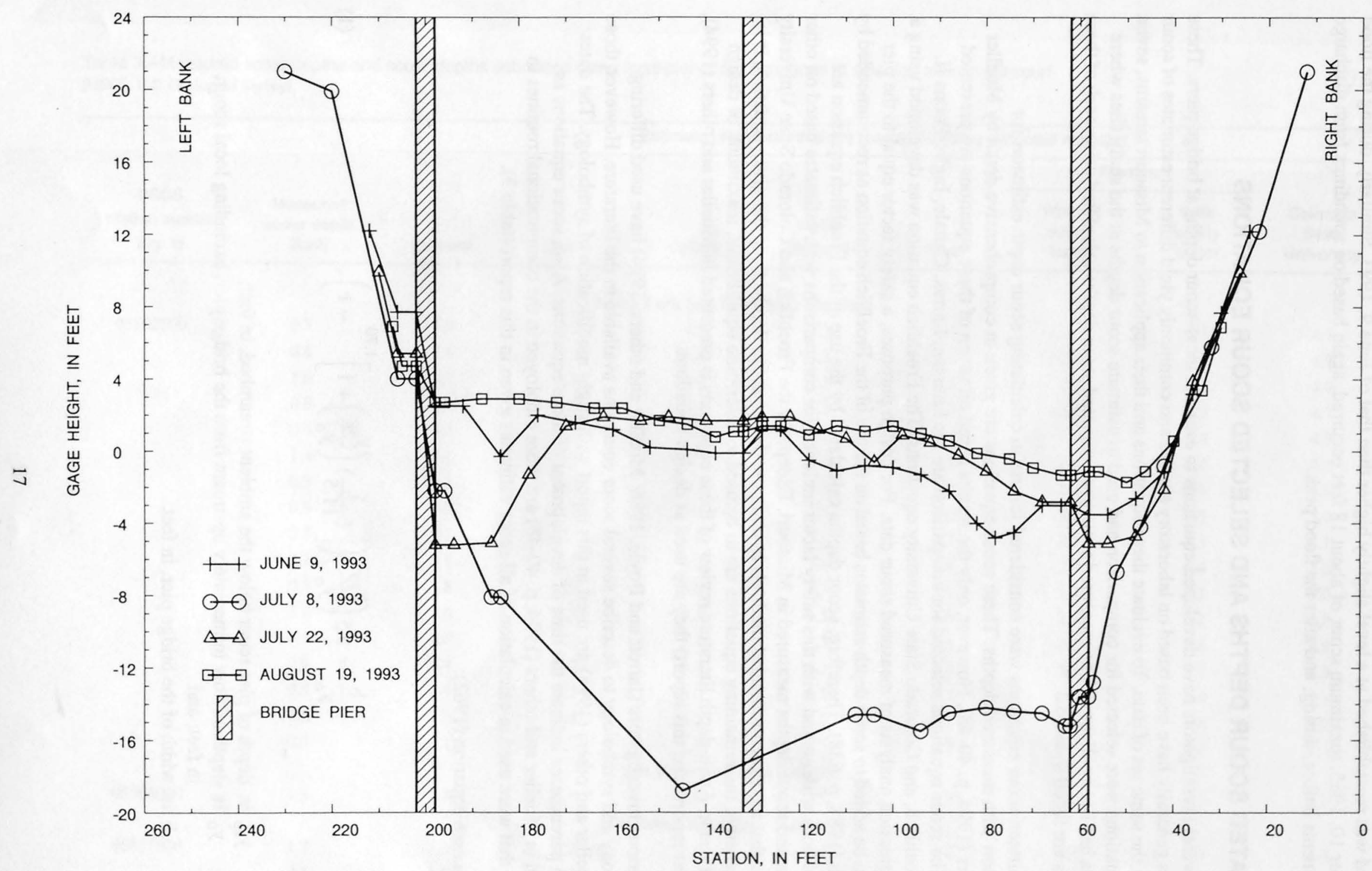

Figure 9. Streambed cross sections at the Chariton River near Prairie Hill, Missouri (station number 06905500; soundings along downstream side of bridge). 
streambed was reestablished at a level slightly higher than that of June 9, 1993. Similarly, during the flood of October 10,1985, maximum scour of about 18 feet occurred, again based on soundings from discharge measurements before, during, and after the flood peak.

\section{ESTIMATED SCOUR DEPTHS AND SELECTED SCOUR EQUATIONS}

Several investigators have developed equations to estimate local-scour depths at bridge piers. These equations generally have been based on laboratory studies and commonly yield different estimates of scour depth for the same set of data. To evaluate these equations and their application to Missouri streams, seven scour equations were selected for comparison and used to estimate scour depths at the study sites where scour data had been collected. The measured scour and estimated scour depths calculated using each of these equations are listed in table 3.

Thirteen scour equations were considered for use in calculating scour depth estimates for comparison with measured depths. These scour equations are given in comprehensive detail by Mueller and others (1994, p. 40-48). However, only the results based on seven of these equations are presented herein. The scour equations selected for comparison are the Laursen, Larras, Chitale, Inglis-Poona II, Shen, Froehlich, and Colorado State University equations. The Froehlich equation was developed using a linear regression analysis of measured scour data. For design purposes, a safety factor equal to the pier width can be added to scour depth estimates based on the use of the Froehlich equation as recommended by Froehlich (1988, p. 538). Therefore, scour depths calculated by the use of the Froehlich equation are presented both without and with this safety factor included for comparisons with estimates based on other equations and scour depths measured in Missouri. Except for the Froehlich and Colorado State University equations that have recommended methods to compute design scour depths from the given equation, it is unclear whether the remaining equations are to be used to determine equilibrium, maximum, or design depths of scour. An in-depth literature review of these equations is presented in Mueller and others (1994), and in that report (and this report) they are used as design equations.

Various investigators (Jarrett and Boyle, 1986; Mueller and others, 1994) have used differing terminology and symbology to describe several scour equations available in the literature. However, those from Mueiler and others (1994) are used in this report with slight modification of symbology. The dates shown in parentheses indicate the time of development of these equations. Also, scour equations as presented in Mueller and others (1994, p. 40-48) are those employed in the computational routines in BSDMS that were used in calculation of all scour estimates given in this report (table 3 ).

Laursen equation (1962):

$$
\frac{\mathrm{b}}{\mathrm{y}_{\mathrm{o}}}=5.5\left(\frac{\mathrm{y}_{\mathrm{sp}}}{\mathrm{y}_{\mathrm{o}}}\right)\left(\left[\left(\frac{1}{11.5}\right)\left(\frac{\mathrm{y}_{\mathrm{sp}}}{\mathrm{y}_{\mathrm{o}}}\right)+1\right]^{1.70}-1\right),
$$

where

$\mathrm{y}_{\mathrm{sp}}$ is depth of pier scour below the ambient streambed, in feet;

$\mathrm{y}_{\mathrm{o}}$ is depth of flow immediately upstream from the bridge pier, excluding local scour, in feet; and

$\mathrm{b}$ is width of the bridge pier, in feet. 
Table 3.-Measured scour depths and scour depths estimated using local-pier-scour equations at selected sites in Missouri [USGS, U.S. Geological Survey]

\begin{tabular}{|c|c|c|c|c|c|c|c|c|c|}
\hline \multirow[b]{3}{*}{$\begin{array}{c}\text { USGS } \\
\text { station number } \\
\text { (fig. 1) }\end{array}$} & \multirow[b]{3}{*}{$\begin{array}{l}\text { Measured } \\
\text { scour depth } \\
\text { (feet) }\end{array}$} & \multicolumn{8}{|c|}{ Estimated scour depth (feet) calculated using indicated equation } \\
\hline & & \multirow[b]{2}{*}{ Laursen } & \multirow[b]{2}{*}{ Larras } & \multirow[b]{2}{*}{ Chitale } & \multirow[b]{2}{*}{$\begin{array}{l}\text { Inglis- } \\
\text { Poona II }\end{array}$} & \multirow[b]{2}{*}{ Shen } & \multicolumn{2}{|c|}{ Froehlich } & \multirow[b]{2}{*}{$\begin{array}{c}\text { Colorado } \\
\text { State } \\
\text { University }\end{array}$} \\
\hline & & & & & & & $\begin{array}{l}\text { Without } \\
\text { safety } \\
\text { factor }\end{array}$ & $\begin{array}{l}\text { With } \\
\text { safety } \\
\text { factor }\end{array}$ & \\
\hline \multicolumn{10}{|c|}{ North Fork Salt River near Shelbina } \\
\hline \multirow[t]{17}{*}{05502500} & 0.7 & 6.0 & 3.2 & 4.3 & 3.3 & 3.3 & 2.3 & 5.3 & 4.7 \\
\hline & 0.6 & 5.8 & 3.2 & 4.4 & 3.3 & 3.3 & 2.2 & 5.2 & 4.7 \\
\hline & 1.0 & 5.7 & 3.2 & 4.4 & 3.3 & 3.3 & 2.2 & 5.2 & 4.7 \\
\hline & 0.9 & 5.9 & 3.2 & 4.4 & 3.3 & 3.3 & 2.3 & 5.3 & 4.7 \\
\hline & 0.5 & 6.3 & 3.2 & 4.8 & 3.3 & 3.4 & 2.4 & 5.4 & 4.9 \\
\hline & 1.4 & 6.1 & 3.2 & 4.5 & 3.3 & 3.4 & 2.3 & 5.3 & 4.8 \\
\hline & 0.9 & 6.2 & 3.2 & 4.5 & 3.3 & 3.4 & 2.3 & 5.3 & 4.8 \\
\hline & 0.8 & 5.8 & 3.2 & 5.5 & 3.3 & 3.6 & 2.3 & 5.3 & 5.0 \\
\hline & 0.8 & 5.7 & 3.2 & 5.9 & 3.3 & 3.7 & 2.3 & 5.3 & 5.1 \\
\hline & 1.1 & 5.9 & 3.2 & 6.5 & 3.3 & 3.8 & 2.4 & 5.4 & 5.2 \\
\hline & 0.8 & 5.8 & 3.2 & 5.5 & 3.3 & 3.6 & 2.3 & 5.3 & 5.0 \\
\hline & 1.2 & 5.6 & 3.2 & 5.1 & 3.3 & 3.5 & 2.2 & 5.2 & 4.8 \\
\hline & 0.8 & 6.0 & 3.2 & 5.1 & 3.3 & 3.5 & 2.3 & 5.3 & 4.9 \\
\hline & 0.9 & 6.4 & 3.2 & 6.8 & 3.3 & 3.8 & 2.5 & 5.5 & 5.3 \\
\hline & 0.9 & 5.9 & 3.2 & 6.0 & 3.3 & 3.7 & 2.4 & 5.4 & 5.1 \\
\hline & 1.0 & 6.2 & 3.2 & 6.1 & 3.3 & 3.7 & 2.4 & 5.4 & 5.2 \\
\hline & 0.7 & 6.3 & 3.2 & 6.1 & 3.3 & 3.7 & 2.4 & 5.4 & 5.2 \\
\hline \multicolumn{10}{|c|}{ Salt River near New London } \\
\hline \multirow[t]{2}{*}{05508000} & 1.3 & 5.2 & 3.6 & 4.8 & 3.5 & 3.9 & 1.7 & 5.2 & 5.1 \\
\hline & \multicolumn{9}{|c|}{ Nodaway River near Skidmore } \\
\hline \multirow[t]{6}{*}{06817600} & 1.7 & 5.2 & 3.2 & 7.4 & 3.2 & 4.2 & 2.3 & 5.3 & 5.4 \\
\hline & 2.1 & 5.2 & 3.2 & 7.4 & 3.2 & 4.2 & 2.3 & 5.3 & 5.4 \\
\hline & 2.5 & 5.6 & 3.2 & 7.6 & 3.3 & 4.1 & 2.4 & 5.4 & 5.4 \\
\hline & 2.1 & 6.7 & 3.2 & 3.9 & 3.2 & 3.3 & 2.5 & 5.5 & 4.8 \\
\hline & 1.8 & 6.6 & 3.2 & 3.9 & 3.2 & 3.3 & 2.5 & 5.5 & 4.8 \\
\hline & 3.3 & 6.2 & 3.2 & 10.1 & 3.3 & 4.6 & 2.7 & 5.7 & 6.0 \\
\hline
\end{tabular}


Table 3.-Measured scour depths and scour depths estimated using local-pier-scour equations at selected sites in Missouri-Continued

\begin{tabular}{|c|c|c|c|c|c|c|c|c|c|}
\hline \multirow[b]{3}{*}{$\begin{array}{l}\text { USGS } \\
\text { station number } \\
\text { (fig. 1) }\end{array}$} & \multirow[b]{3}{*}{$\begin{array}{l}\text { Measured } \\
\text { scour depth } \\
\text { (feet) }\end{array}$} & \multicolumn{8}{|c|}{ Estimated scour depth (feet) calculated using indicated equation } \\
\hline & & \multirow[b]{2}{*}{ Laursen } & \multirow[b]{2}{*}{ Larras } & \multirow[b]{2}{*}{ Chitale } & \multirow[b]{2}{*}{$\begin{array}{l}\text { Inglis- } \\
\text { Poona II }\end{array}$} & \multirow[b]{2}{*}{ Shen } & \multicolumn{2}{|c|}{ Froehlich } & \multirow[b]{2}{*}{$\begin{array}{c}\text { Colorado } \\
\text { State } \\
\text { University }\end{array}$} \\
\hline & & & & & & & $\begin{array}{l}\text { Without } \\
\text { safety } \\
\text { factor }\end{array}$ & $\begin{array}{l}\text { With } \\
\text { safety } \\
\text { factor }\end{array}$ & \\
\hline \multicolumn{10}{|c|}{ Grand River near Sumner } \\
\hline \multirow[t]{3}{*}{06902000} & 7.1 & 11.5 & 4.6 & 11.5 & 4.9 & 6.0 & 5.2 & 10.0 & 8.9 \\
\hline & 6.6 & 12.1 & 4.5 & 9.3 & 4.3 & 5.6 & 5.2 & 9.9 & 8.6 \\
\hline & 6.5 & 11.9 & 4.5 & 8.4 & 4.4 & 5.4 & 5.1 & 9.8 & 8.4 \\
\hline \multicolumn{10}{|c|}{ Chariton River at Novinger } \\
\hline \multirow[t]{3}{*}{06904500} & 1.9 & 5.7 & 4.6 & 5.0 & 4.1 & 4.9 & 2.7 & 7.5 & 6.4 \\
\hline & 3.4 & 5.9 & 4.5 & 3.6 & 4.2 & 4.1 & 2.6 & 7.2 & 5.7 \\
\hline & 1.9 & 8.5 & 4.2 & 7.7 & 4.6 & 4.9 & 3.5 & 7.7 & 7.0 \\
\hline \multicolumn{10}{|c|}{ Chariton River near Prairie Hill } \\
\hline \multirow[t]{2}{*}{06905500} & 3.2 & 9.3 & 3.9 & 11.3 & 4.0 & 5.3 & 3.9 & 7.8 & 7.6 \\
\hline & 4.7 & 11.2 & 4.1 & 11.8 & 3.3 & 5.5 & 4.5 & 8.6 & 8.2 \\
\hline \multicolumn{10}{|c|}{ Little Piney Creek at Newburg } \\
\hline \multirow[t]{12}{*}{06932000} & 0.7 & 5.6 & 3.7 & 9.8 & 3.7 & 5.6 & 2.6 & 6.2 & 6.8 \\
\hline & 1.1 & 6.0 & 3.7 & 10.6 & 3.8 & 5.6 & 2.7 & 6.3 & 6.9 \\
\hline & 1.5 & 6.5 & 3.6 & 14.2 & 3.8 & 6.3 & 3.0 & 6.5 & 7.7 \\
\hline & 2.4 & 6.8 & 3.6 & 15.0 & 3.9 & 6.3 & 3.1 & 6.6 & 7.7 \\
\hline & 2.4 & 6.9 & 3.6 & 16.3 & 3.7 & 6.4 & 3.1 & 6.5 & 7.9 \\
\hline & 1.8 & 7.3 & 3.6 & 17.5 & 3.7 & 6.5 & 3.3 & 6.7 & 8.0 \\
\hline & 1.4 & 6.9 & 3.7 & 15.0 & 4.0 & 6.4 & 3.1 & 6.7 & 7.9 \\
\hline & 0.8 & 6.4 & 3.6 & 5.7 & 3.8 & 4.0 & 2.5 & 6.0 & 5.6 \\
\hline & 1.7 & 6.0 & 3.6 & 4.8 & 3.7 & 3.7 & 2.4 & 5.8 & 5.2 \\
\hline & 0.7 & 6.9 & 3.6 & 11.6 & 3.7 & 5.2 & 2.9 & 6.3 & 6.8 \\
\hline & 1.0 & 6.8 & 3.6 & 11.2 & 3.7 & 5.2 & 2.9 & 6.3 & 6.7 \\
\hline & 0.9 & 6.4 & 3.6 & 5.7 & 3.8 & 4.0 & 2.5 & 6.0 & 5.6 \\
\hline \multicolumn{10}{|c|}{ Gasconade River near Vienna } \\
\hline \multirow[t]{3}{*}{06933850} & 0.7 & 7.2 & 3.6 & 4.1 & 3.8 & 3.6 & 2.1 & 5.6 & 5.4 \\
\hline & 1.0 & 7.5 & 3.6 & 3.3 & 3.6 & 3.5 & 2.1 & 5.5 & 5.2 \\
\hline & 1.9 & 7.5 & 3.6 & 3.3 & 3.6 & 3.5 & 2.1 & 5.5 & 5.2 \\
\hline
\end{tabular}


Table 3.-Measured scour depths and scour depths estimated using local-pier-scour equations at selected sites in Missouri-Continued

\begin{tabular}{|c|c|c|c|c|c|c|c|c|c|}
\hline \multirow[b]{3}{*}{$\begin{array}{c}\text { USGS } \\
\text { station number } \\
\text { (fig. 1) }\end{array}$} & \multirow[b]{3}{*}{$\begin{array}{l}\text { Measured } \\
\text { scour depth } \\
\text { (feet) }\end{array}$} & \multicolumn{8}{|c|}{ Estimated scour depth (feet) calculated using indicated equation } \\
\hline & & \multirow[b]{2}{*}{ Laursen } & \multirow[b]{2}{*}{ Larras } & \multirow[b]{2}{*}{ Chitale } & \multirow[b]{2}{*}{$\begin{array}{l}\text { Inglis- } \\
\text { Poona II }\end{array}$} & \multirow[b]{2}{*}{ Shen } & \multicolumn{2}{|c|}{ Froehlich } & \multirow[b]{2}{*}{$\begin{array}{c}\text { Colorado } \\
\text { State } \\
\text { University }\end{array}$} \\
\hline & & & & & & & $\begin{array}{l}\text { Without } \\
\text { safety } \\
\text { factor }\end{array}$ & $\begin{array}{l}\text { With } \\
\text { safety } \\
\text { factor }\end{array}$ & \\
\hline \multicolumn{10}{|c|}{ Gasconade River near Rich Fountain } \\
\hline \multirow{3}{*}{06934000} & 3.5 & 12.0 & 4.4 & 10.1 & 3.9 & 5.6 & 3.6 & 8.1 & 8.5 \\
\hline & 2.8 & 9.7 & 4.7 & 17.0 & 5.5 & 7.6 & 3.5 & 8.5 & 10.0 \\
\hline & 4.1 & 11.7 & 4.4 & 27.4 & 4.1 & 8.3 & 4.0 & 8.5 & 11.1 \\
\hline \multicolumn{10}{|c|}{ Big River at Irondale } \\
\hline \multirow[t]{4}{*}{07017200} & 1.2 & 4.6 & 5.0 & 4.1 & 3.4 & 5.4 & 1.9 & 7.3 & 6.5 \\
\hline & 1.3 & 6.5 & 4.9 & 10.0 & 4.7 & 7.3 & 2.7 & 7.9 & 8.8 \\
\hline & 1.7 & 4.7 & 5.0 & 4.2 & 3.5 & 5.3 & 1.9 & 7.3 & 6.5 \\
\hline & 1.7 & 4.4 & 5.0 & 3.4 & 3.3 & 4.9 & 1.7 & 7.1 & 6.0 \\
\hline
\end{tabular}


Laursen found that the most important aspect of the geometry of the pier was the angle of attack between the pier and the flow, coupled with the length-width ratio of the pier. The shape of the pier also is important if the pier is aligned with the flow. Therefore, the depth of scour from equation 1 must be corrected for pier shape if the pier is aligned with the flow

$$
\mathrm{y}_{\mathrm{sp}}=\mathrm{K}_{\mathrm{S} 1} \mathrm{y}_{\mathrm{sp}} \text {, }
$$

and, for angle of attack if the pier is not aligned with the flow,

$$
\mathrm{y}_{\mathrm{sp}}=\mathrm{K}_{\alpha \mathrm{L}} \mathrm{y}_{\mathrm{sp}}
$$

where

$\mathrm{y}_{\mathrm{sp}}$ is depth of pier scour below the ambient streambed, in feet;

$\mathrm{K}_{\alpha \mathrm{L}}$ is a coefficient based on the angle of the approach flow referenced to the bridge pier (fig. 10); and

$\mathrm{K}_{\mathrm{S} 1}$ is a coefficient based on the shape of the pier nose (table 4).

Larras equation (1963):

$$
\mathrm{y}_{\mathrm{sp}}=1.42 \mathrm{~K}_{\mathrm{S} 2} \mathrm{~b}^{0.75}
$$

where

$\mathrm{y}_{\text {sp }}$ is depth of pier scour below the ambient streambed, in feet;

$\mathrm{b}$ is width of the bridge pier, in feet; and

$\mathrm{K}_{\mathrm{S} 2}$ is a coefficient based on the shape of the pier nose (1.0 for cylindrical piers and 1.4 for rectangular piers).

Chitale equation (1962):

$$
y_{s p}=y_{o}\left(-5.49 F_{o}^{2}+6.65 F_{o}-0.51\right)
$$

where

$y_{s p}$ is depth of pier scour below the ambient streambed, in feet;

$y_{0}$ is depth of flow immediately upstream from the bridge pier or abutment, excluding local scour, in feet; and

where

$$
F_{\mathrm{o}}=\frac{\mathrm{V}_{\mathrm{o}}}{\sqrt{\mathrm{gy}_{\mathrm{o}}}}
$$

$\boldsymbol{F}_{\mathrm{O}}$ is the Froude number of the flow immediately upstream from the pier;

$\mathrm{V}_{\mathrm{O}}$ is velocity of the approach flow immediately upstream from the bridge pier or abutment; and

$\mathrm{g}$ is the acceleration of gravity. 


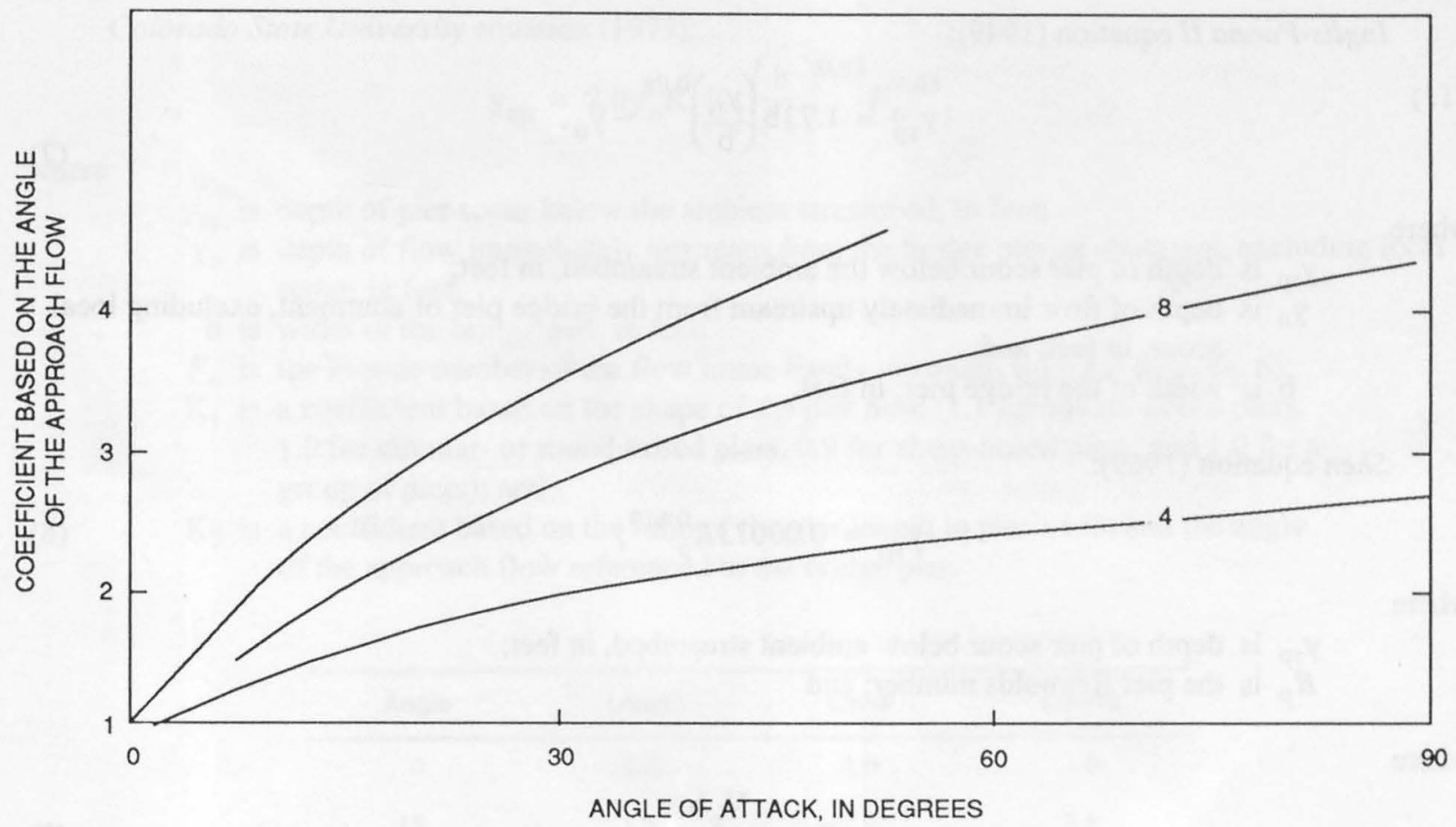

Figure 10. Effect of angle of attack on bridge pier (modified from Laursen, 1962, p. 177).

Table 4. Pier-shape coefficients (Laursen equation)

$\left[K_{s 1}\right.$, shape coefficients for nose forms (to be used only for piers aligned with flow); modified from Laursen, 1962, p. 177]

$\mathrm{K}_{\mathrm{s} 1}$

Rectangle

Not applicable

Semicircular

Not applicable

Elliptic

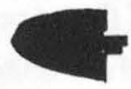


Inglis-Poona II equation (1949):

$$
y_{s p}=1.73 b\left(\frac{y_{0}}{b}\right)^{0.78}-y_{o},
$$

where

$\mathrm{y}_{\mathrm{sp}}$ is depth of pier scour below the ambient streambed, in feet;

$y_{0}$ is depth of flow immediately upstream from the bridge pier or abutment, excluding local scour, in feet; and

b is width of the bridge pier, in feet.

Shen equation (1969):

$$
\mathrm{y}_{\mathrm{sp}}=0.00073 R_{\mathrm{p}}^{0.619},
$$

where

$\mathrm{y}_{\mathrm{sp}}$ is depth of pier scour below ambient streambed, in feet;

$\boldsymbol{R}_{\mathrm{p}}$ is the pier Reynolds number; and

where

$$
\boldsymbol{R}_{\mathrm{p}}=\frac{\mathrm{V}_{\mathrm{o}} \mathrm{b}}{\mathrm{v}},
$$

$\mathrm{V}_{\mathrm{O}}$ is velocity of the approach flow immediately upstream from the bridge pier or abutment, in feet per second;

$\mathrm{b}$ is width of the bridge pier, in feet; and

$v$ is the kinematic viscosity of water.

Froehlich equation (1988):

$$
\mathrm{y}_{\mathrm{sp}}=0.32 \mathrm{~b} \phi\left(\frac{\mathrm{b}^{\prime}}{\mathrm{b}}\right)^{0.62}\left(\frac{\mathrm{y}_{0}}{\mathrm{~b}}\right)^{0.46} F_{\mathrm{o}}^{0.2}\left(\frac{\mathrm{b}}{\mathrm{d}_{50}}\right)^{0.08},
$$

where

$\mathrm{y}_{\mathrm{sp}}$ is depth of pier scour below the ambient streambed, in feet;

$\mathrm{y}_{0}$ is depth of flow immediately upstream from the bridge pier or abutment, excluding local scour, in feet;

$\mathrm{b}$ is width of the bridge pier, in feet;

$\phi$ is a coefficient based on the shape of the pier nose (1.3 for square-nosed piers,

1.0 for round-nosed piers, and 0.7 for sharp-nosed piers);

$\mathrm{d}_{50}$ is median grain size of the streambed material, in feet;

$b^{\prime}$ is width of the bridge pier projected normal to the approach flow, in feet;

where

$\mathrm{b}^{\prime}=b \cos (\alpha)+L \sin (\alpha)$

$\alpha$ is angle of the approach flow referenced to the bridge pier, in degrees; and

$L$ is length of the bridge pier, in feet.

and

$F_{\mathrm{o}}$ is the Froude number of the flow immediately upstream from the pier (eq. 6). 
Colorado State University equation (1975):

$$
\mathrm{y}_{\mathrm{sp}}=2.0 \mathrm{y}_{\mathrm{o}} \mathrm{K}_{1} \mathrm{~K}_{2}\left(\frac{\mathrm{b}}{\mathrm{y}_{\mathrm{o}}}\right)^{0.65} F_{\mathrm{o}}^{0.43},
$$

where

$\mathrm{y}_{\mathrm{sp}}$ is depth of pier scour below the ambient streambed, in feet;

$y_{o}$ is depth of flow immediately upstream from the bridge pier or abutment, excluding local scour, in feet;

$\mathrm{b}$ is width of the bridge pier, in feet;

$\boldsymbol{F}_{\mathrm{o}}$ is the Froude number of the flow immediately upstream from the pier (eq. 6);

$\mathrm{K}_{1}$ is a coefficient based on the shape of the pier nose (1.1 for square-nosed piers, 1.0 for circular- or round-nosed piers, 0.9 for sharp-nosed piers, and 1.0 for a group of piers); and

$\mathrm{K}_{2}$ is a coefficient based on the ratio of the pier length to pier width and the angle of the approach flow referenced to the bridge pier.

\begin{tabular}{cccc}
\hline Angle & $\mathrm{L} / \mathrm{b}=\mathbf{4}$ & $\mathrm{L} / \mathrm{b}=\mathbf{8}$ & $\mathrm{L} / \mathrm{b}=\mathbf{1 2}$ \\
\hline 0 & 1.0 & 1.0 & 1.0 \\
15 & 1.5 & 2.0 & 2.5 \\
30 & 2.0 & 2.5 & 3.5 \\
45 & 2.3 & 3.3 & 4.3 \\
90 & 2.5 & 3.9 & 5.0 \\
\hline
\end{tabular}

Richardson and others (1991) recommend a 10-percent increase to compute maximum scour for both antidunes and plane-bed configurations. This recommendation was used for the computations of scour estimates in this report.

\section{MEASURED AND ESTIMATED SCOUR DEPTHS}

Of the 54 local-scour measurements shown in table 2, statistical tables and graphs are based on 39 measurements. Only the larger measurement was retained in cases where both upstream and downstream measurements were made at the same pier during the same flood. The range of scour hole depths measured at each of the 10 sites is shown in figure 11. A single plotting point may represent multiple occurrences of the same value.

Scour parameters (equation variables) of pier width, mean bed-material diameter, flow depth, and flow velocity were used as ordinate values and the depth was used as the abscissa value for each scour measurement to indicate the relation the scour parameters have with scour depths (figs. 12-15; table 2). Because all piers were considered round-nosed and no appreciable flow angles of attack were experienced at these piers during scour measurements, neither pier shape nor angle of attack was a factor related to measured scour depths. The bed-material samples obtained during this study are assumed to be representative of the bed-material size at the time of the scour measurements. Some piers were tapered from top to bottom resulting in effective pier widths normal to flow that varied with flow depths. For the 10 sites in Missouri, these figures indicate that measured scour depths are not dependent on, nor can they be 


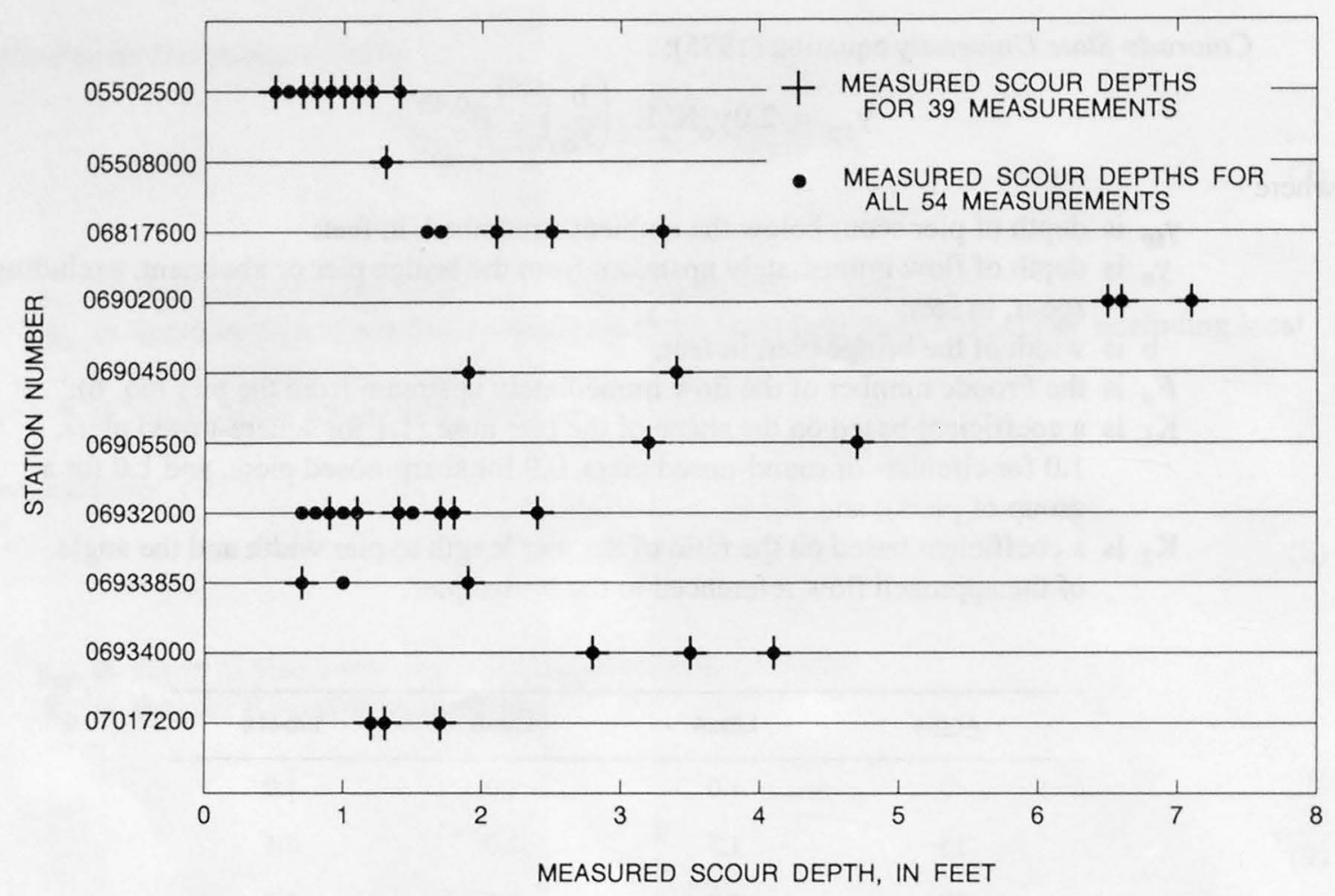

Figure 11. Measured scour depths for each of the selected sites in Missouri.

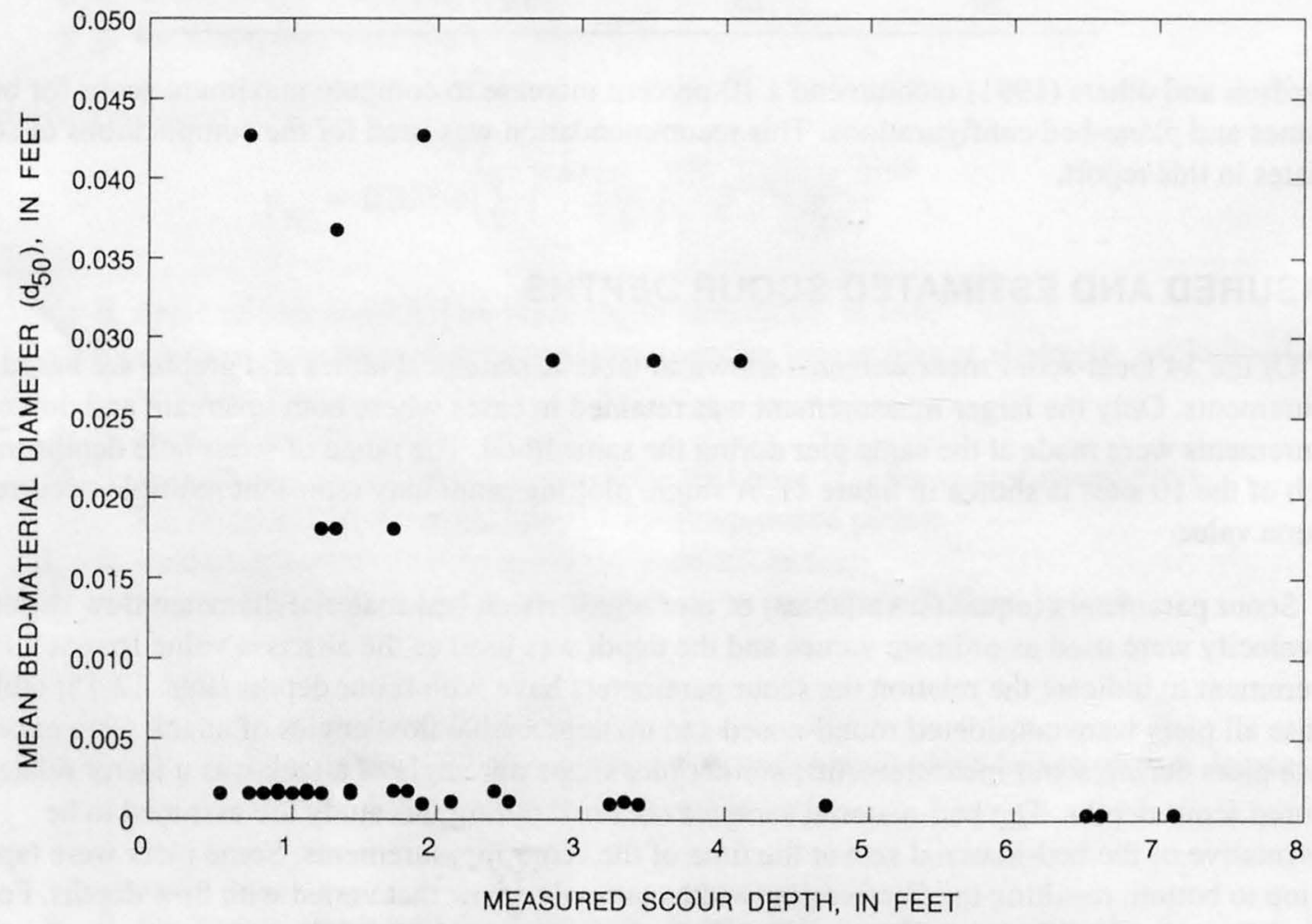

Figure 12. Relation between mean bed-material diameter and measured scour depth at selected sites in Missouri. 


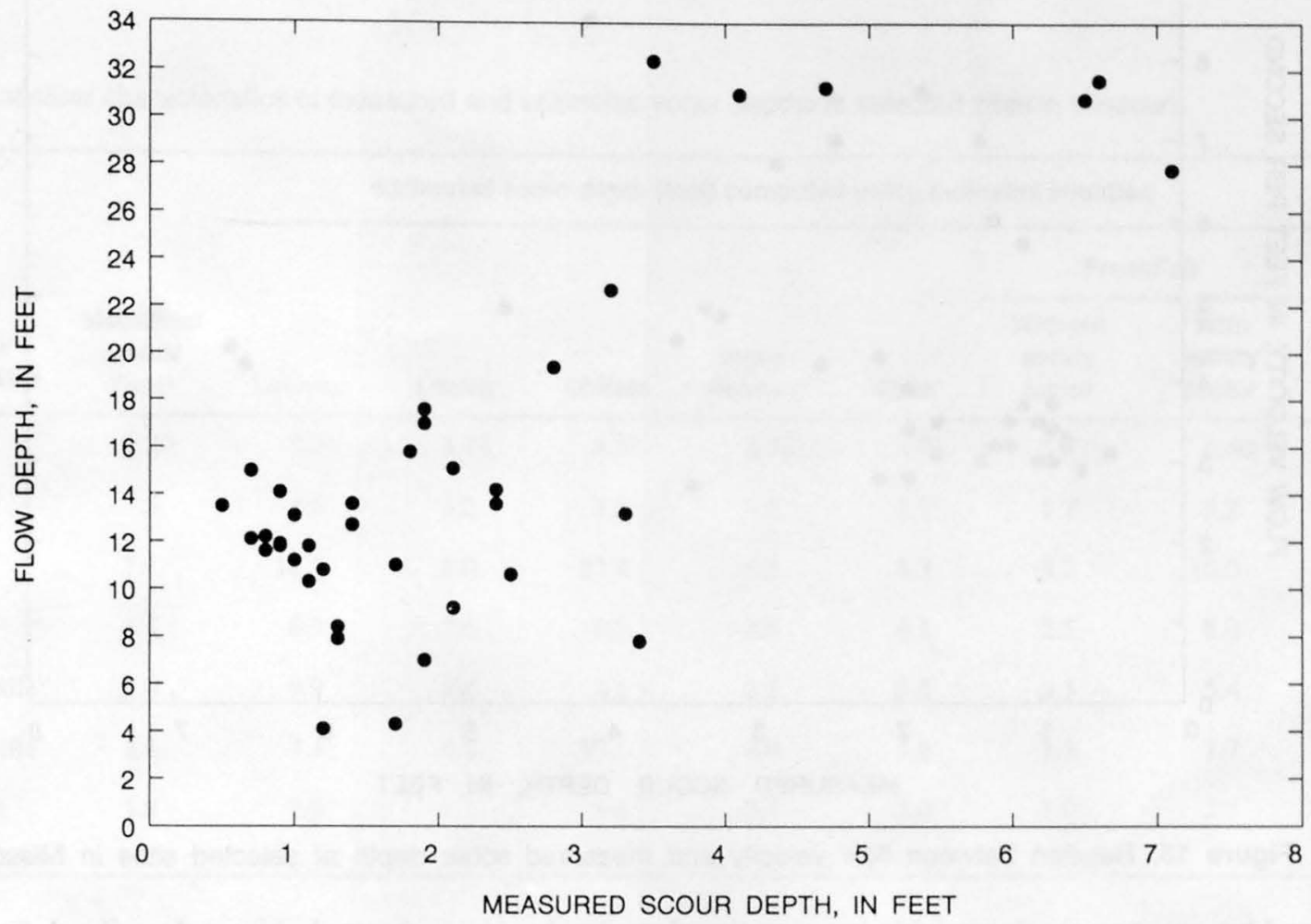

Figure 13. Relation between flow depth and measured scour depth at selected sites in Missouri.

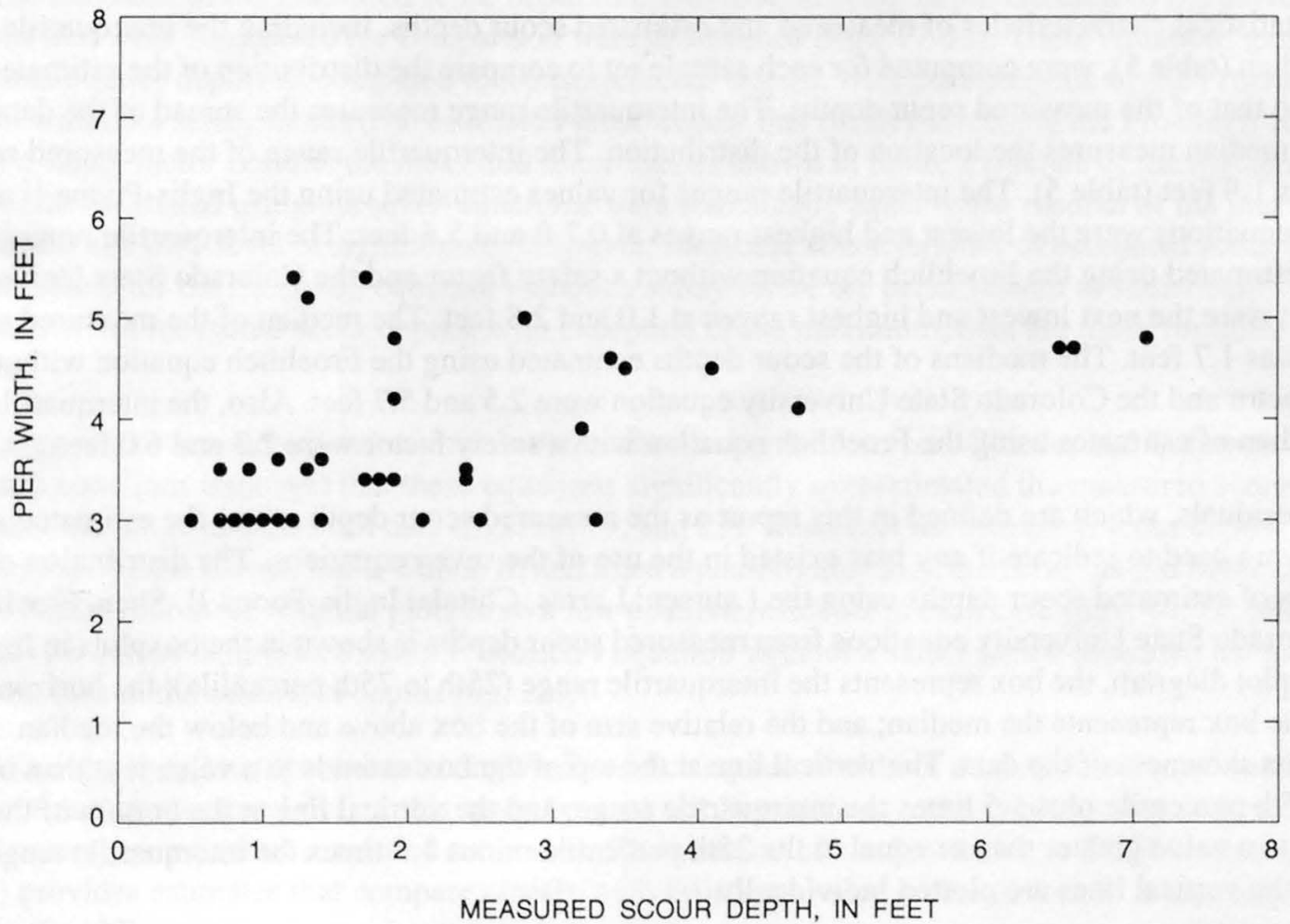

Figure 14. Relation between pier width and measured scour depth at selected sites in Missouri. 


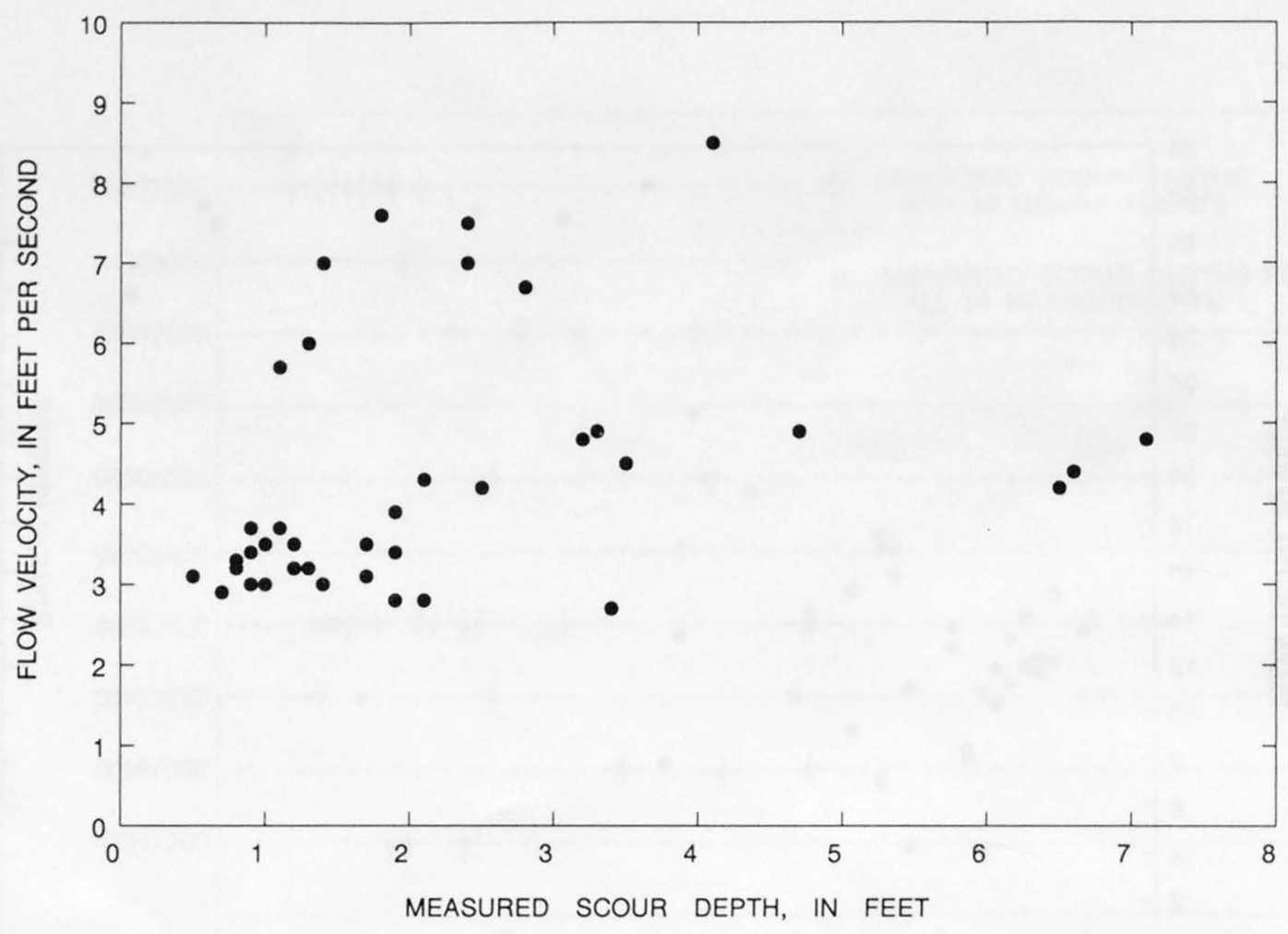

Figure 15. Relation between flow velocity and measured scour depth at selected sites in Missouri.

explained by, any one single variable because the plotted values in each graph do not describe definite, close fitting lines of relation with scour depth. However, flow depth indicated the most significant relation with depth of scour.

Statistical characteristics of measured and estimated scour depths, including the interquartile range and median (table 5), were computed for each sample set to compare the distribution of the estimated scour depths to that of the measured scour depths. The interquartile range measures the spread of the data points and the median measures the location of the distribution. The interquartile range of the measured scour depths is 1.8 feet (table 5). The interquartile ranges for values estimated using the Inglis-Poona II and Chitale equations were the lowest and highest ranges at $0.7 \mathrm{ft}$ and $5.6 \mathrm{feet}$. The interquartile ranges for values estimated using the Froehlich equation without a safety factor and the Colorado State University equation were the next lowest and highest ranges at 1.0 and 2.8 feet. The median of the measured scour depths was 1.7 feet. The medians of the scour depths estimated using the Froehlich equation without a safety factor and the Colorado State University equation were 2.5 and 5.7 feet. Also, the interquartile range and median of estimates using the Froehlich equation with a safety factor were 2.3 and 6.0 feet.

Residuals, which are defined in this report as the measured scour depth minus the estimated scour depth, were used to indicate if any bias existed in the use of the seven equations. The distribution of the residuals of estimated scour depths using the Laursen, Larras, Chitale, Inglis-Poona II, Shen, Froehlich, and Colorado State University equations from measured scour depths is shown in the boxplots in figure 16. In a boxplot diagram, the box represents the interquartile range ( 25 th to 75 th percentile); the horizontal line inside the box represents the median; and the relative size of the box above and below the median represents skewness of the data. The vertical line at the top of the box extends to a value less than or equal to the 75 th percentile plus 1.5 times the interquartile range, and the vertical line at the bottom of the box extends to a value greater than or equal to the 25 th percentile minus 1.5 times the interquartile range. Data beyond the vertical lines are plotted individually. 
Table 5.--Statistical characteristics of measured and estimated scour depths at selected sites in Missouri

\begin{tabular}{|c|c|c|c|c|c|c|c|c|c|}
\hline \multirow[b]{3}{*}{$\begin{array}{c}\text { Statistical } \\
\text { characteristic }\end{array}$} & \multirow[b]{3}{*}{$\begin{array}{l}\text { Measured } \\
\text { scour } \\
\text { (feet) }\end{array}$} & \multicolumn{7}{|c|}{ Estimated scour depth (feet) computed using indicated equation } & \multirow[b]{3}{*}{$\begin{array}{c}\text { Colorado } \\
\text { State } \\
\text { Universiy }\end{array}$} \\
\hline & & \multirow[b]{2}{*}{ Laursen } & \multirow[b]{2}{*}{ Larras } & \multirow[b]{2}{*}{ Chitale } & \multirow[b]{2}{*}{$\begin{array}{l}\text { Inglis- } \\
\text { Poona II }\end{array}$} & \multirow[b]{2}{*}{ Shen } & \multicolumn{2}{|c|}{ Froehlich } & \\
\hline & & & & & & & $\begin{array}{l}\text { Without } \\
\text { safety } \\
\text { factor }\end{array}$ & $\begin{array}{l}\text { With } \\
\text { safety } \\
\text { factor }\end{array}$ & \\
\hline Mean & 2.20 & 7.21 & 3.78 & 8.31 & 3.70 & 4.77 & 2.87 & 6.60 & 6.48 \\
\hline Minimum & .5 & 4.6 & 3.2 & 3.3 & 3.0 & 3.3 & 1.7 & 5.2 & 4.7 \\
\hline Maximum & 7.1 & 12.1 & 5.0 & 27.4 & 5.5 & 8.3 & 5.2 & 10.0 & 11.1 \\
\hline Median & 1.7 & 6.3 & 3.6 & 6.5 & 3.6 & 4.2 & 2.5 & 6.0 & 5.7 \\
\hline 25 th percentile & 1.0 & 5.9 & 3.2 & 4.5 & 3.3 & 3.6 & 2.3 & 5.4 & 5.1 \\
\hline 75th percentile & 2.8 & 7.5 & 4.4 & 10.1 & 4.0 & 5.6 & 3.3 & 7.7 & 7.9 \\
\hline $\begin{array}{l}\text { Interquartile } \\
\text { range }\end{array}$ & 1.8 & 1.6 & 1.2 & 5.6 & 0.7 & 2.0 & 1.0 & 2.3 & 2.8 \\
\hline
\end{tabular}

The residuals of the measured scour depth minus estimated scour depth for each of the seven scour equations that were considered for comparison were determined (figs. 17-23). These equations generally overestimate scour depths as compared to measured scour depths, with the exception of the Froehlich equation without a safety factor. The estimated scour depths that result from using the Froehlich equation without a safety factor best fits the measured scour data as shown in figure 22 . None of the medians of scour depths estimated using the seven equations were statistically equal to the median of the measured scour depths at a 0.05 level of significance. However, statistical characteristics of estimated scour depths given in table 5 for the Froehlich equation without a safety factor are better related to statistical characteristics of measured scour depths, with exception of the maximum, than are those for other equations.

Large negative residuals for estimates determined using the Laursen, Chitale, and Colorado State University equations indicated that these equations significantly overestimated the measured scour depths throughout the range of measured data (figs. 17, 19, and 23). Residuals for estimated scour depths determined using the Larras, Inglis-Poona II, and Shen equations also indicated bias toward overestimation of scour depths, but these residual plots have a few positive residuals in each case (figs. 18, 20, and 21). Residuals for values determined using Froehlich's equation without a safety factor indicated the least significant bias in the estimated depths (fig. 22).

Scour-depth estimates obtained using the Froehlich equation without a safety factor most nearly agree with scour depths measured during this study because the Froehlich equation was developed based on regression analysis of actual measured scour data. The addition of a safety factor to the Froehlich equation (fig. 22) provides estimates that compare closely with estimates based on the Colorado State University equation (fig. 23). 


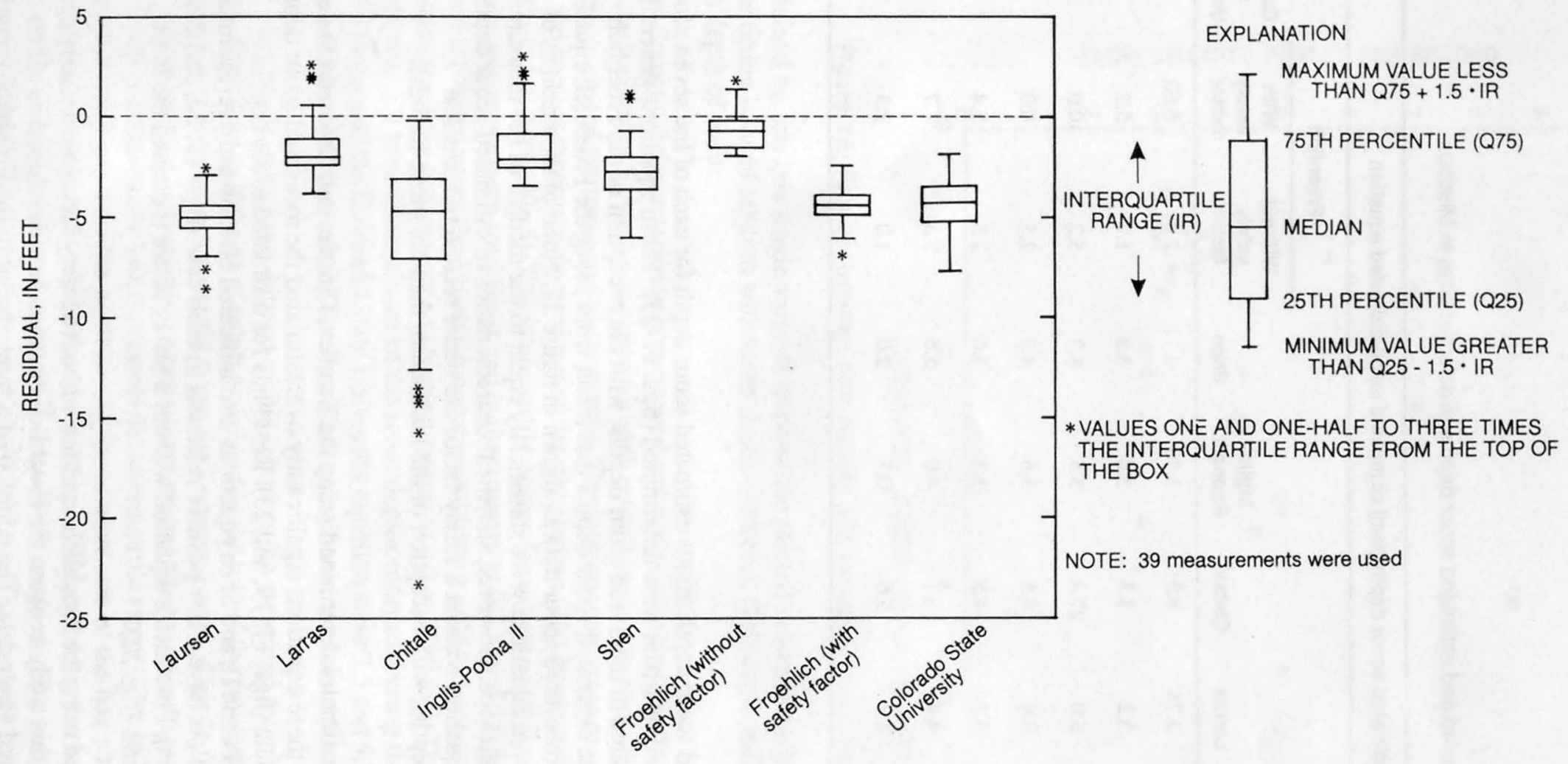

Figure 16. Distribution of residuals of estimated scour depths (using scour equations) from measured scour depths at selected sites in Missouri. 


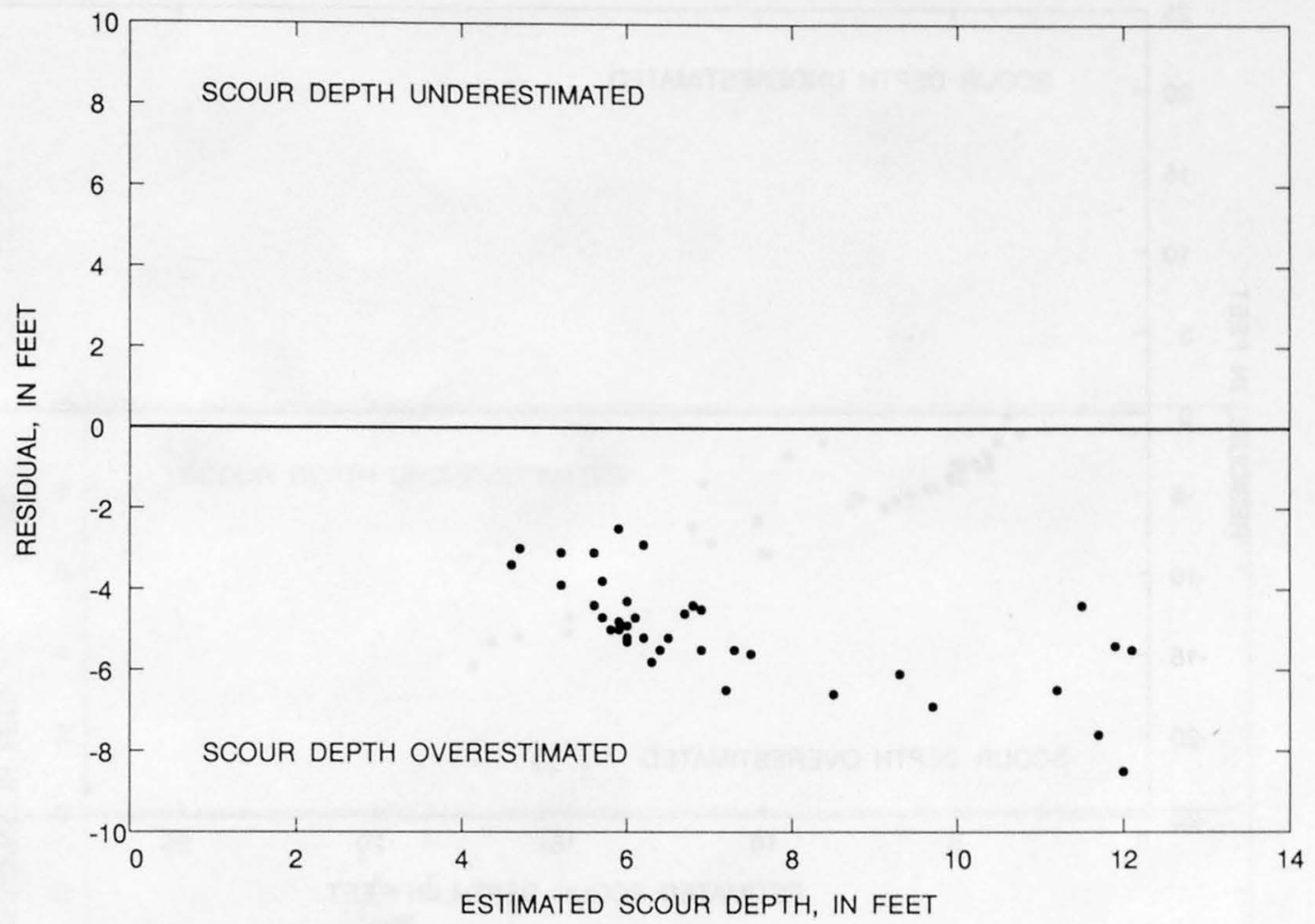

Figure 17. Relation between scour depth estimated using the Laursen equation and the residual (measured scour depth minus the estimated scour depth) at selected sites in Missouri.

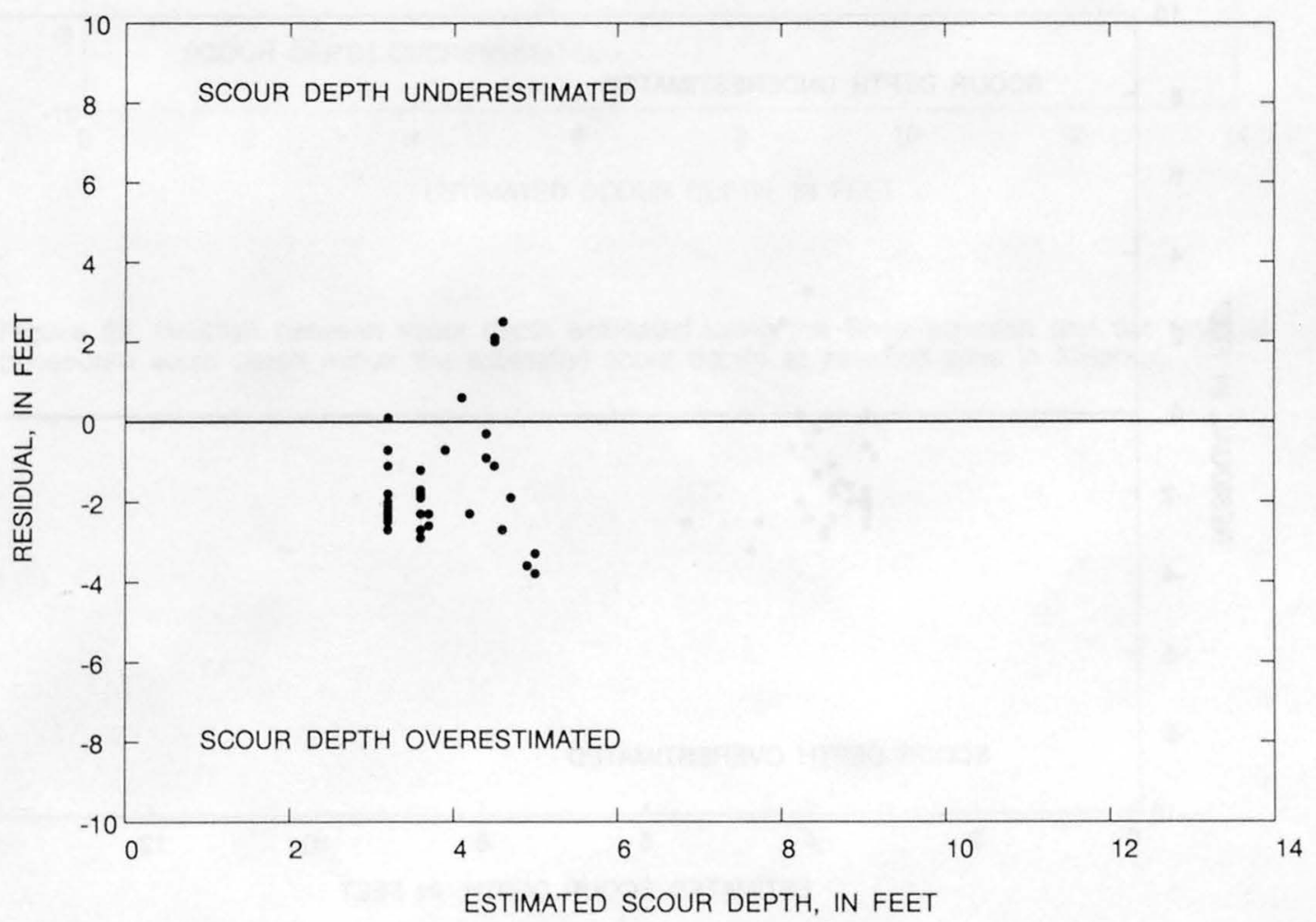

Figure 18. Relation between scour depth estimated using the Larras equation and the residual (measured scour depth minus the estimated scour depth) at selected sites in Missouri. 


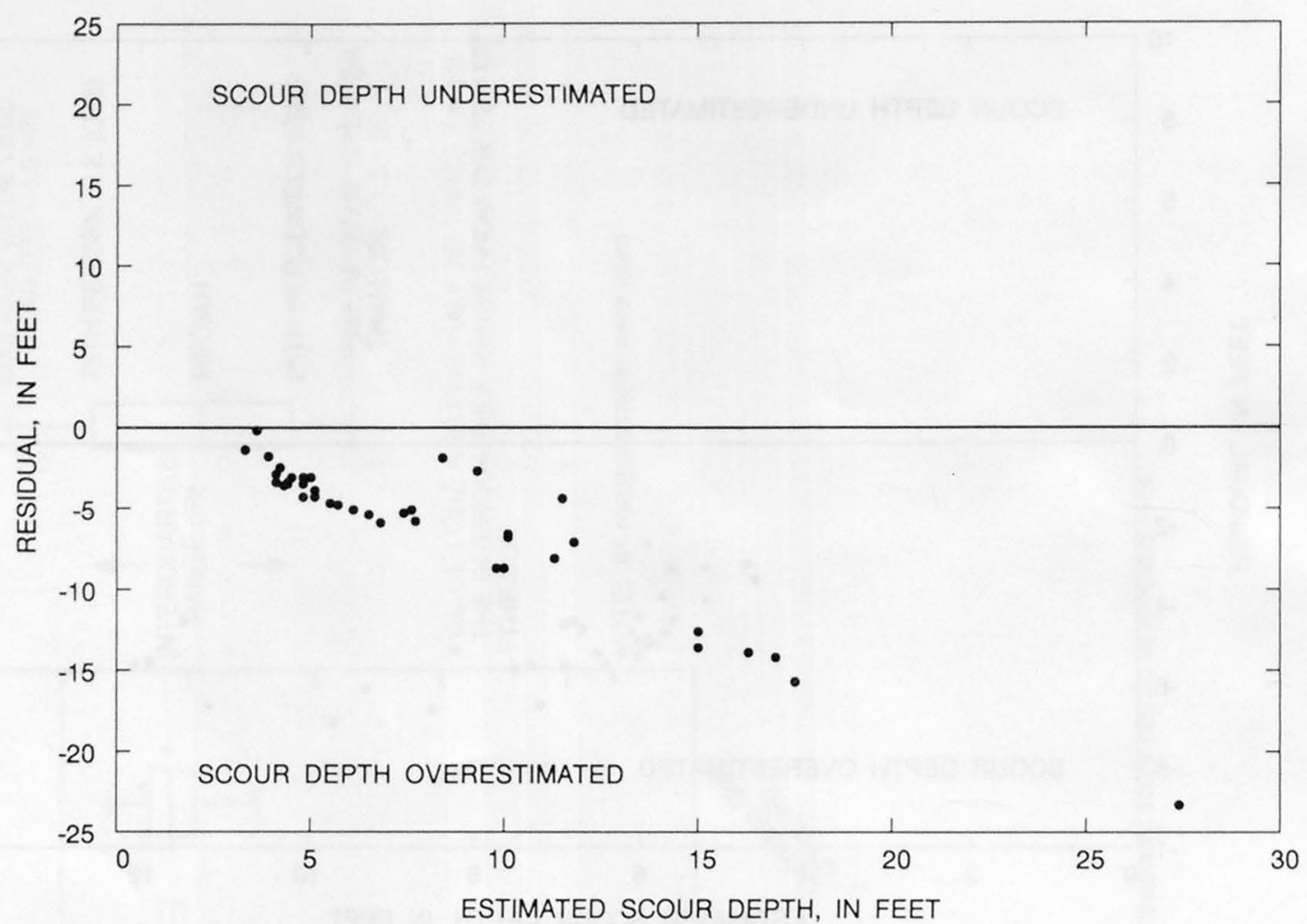

Figure 19. Relation between scour depth estimated using the Chitale equation and the residual (measured scour depth minus the estimated scour depth) at selected sites in Missouri.

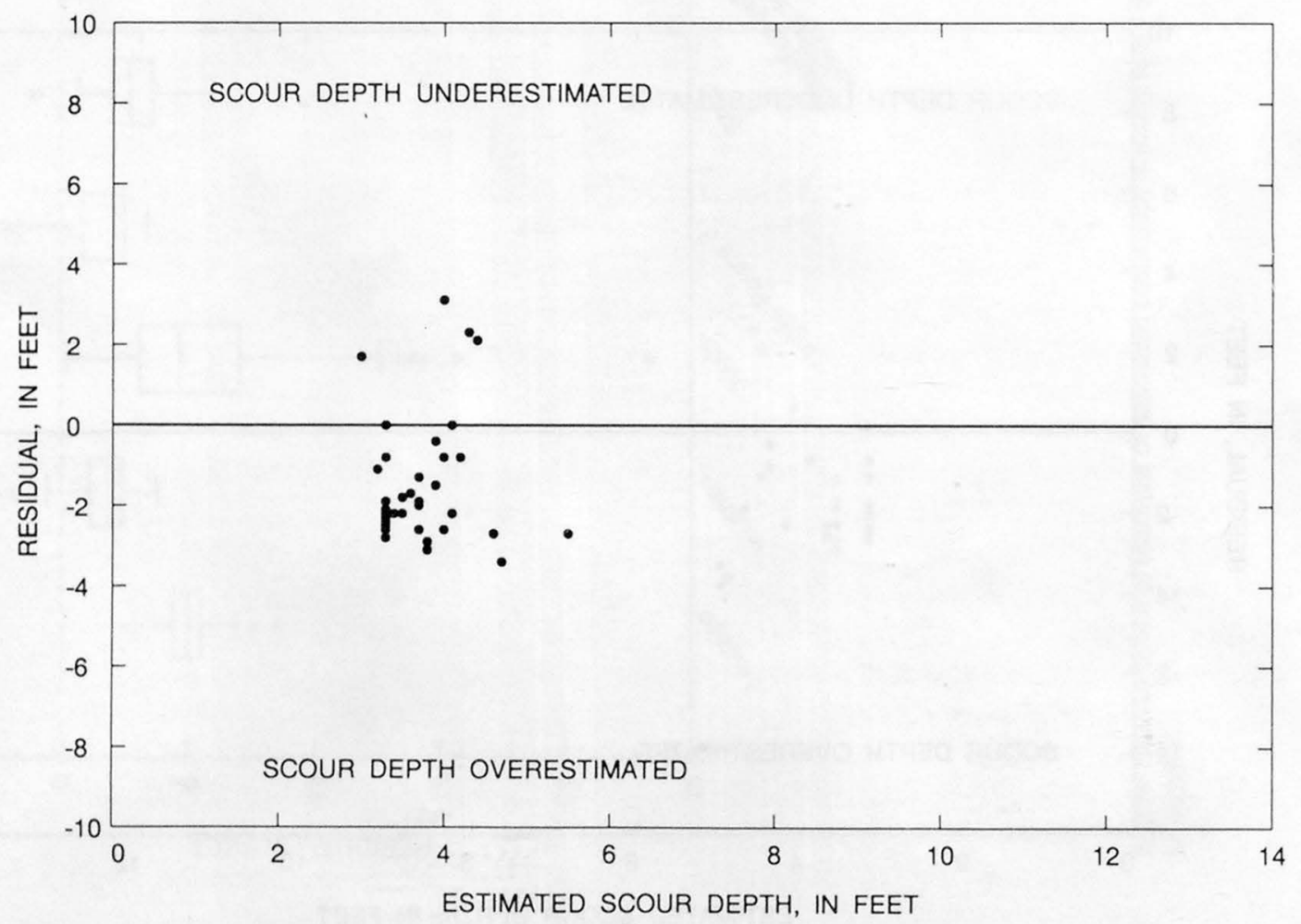

Figure 20. Relation between scour depth estimated using the Inglis-Poona II equation and the residual (measured scour depth minus the estimated scour depth) at selected sites in Missouri. 


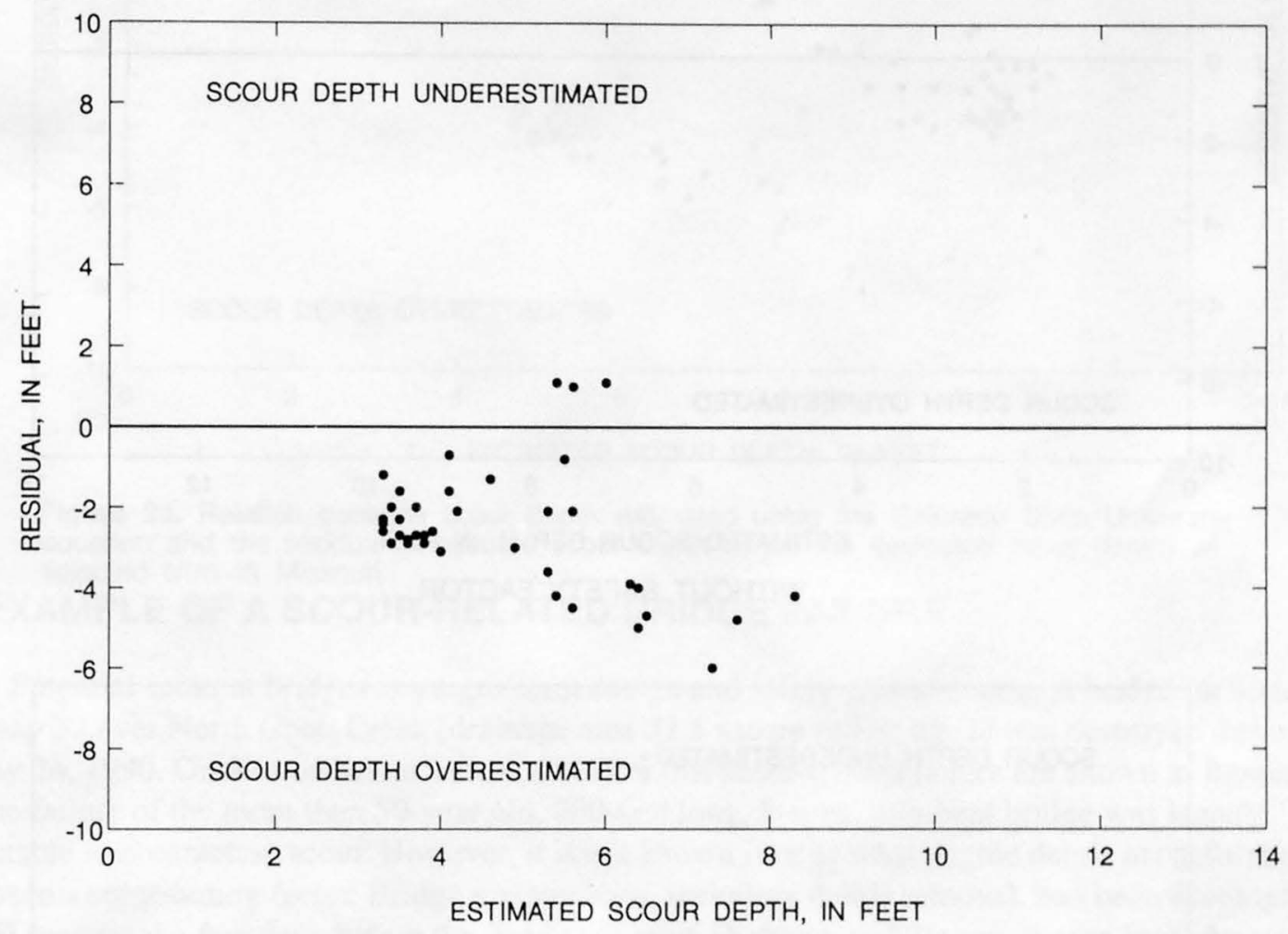

Figure 21. Relation between scour depth estimated using the Shen equation and the residual (measured scour depth minus the estimated scour depth) at selected sites in Missouri. 

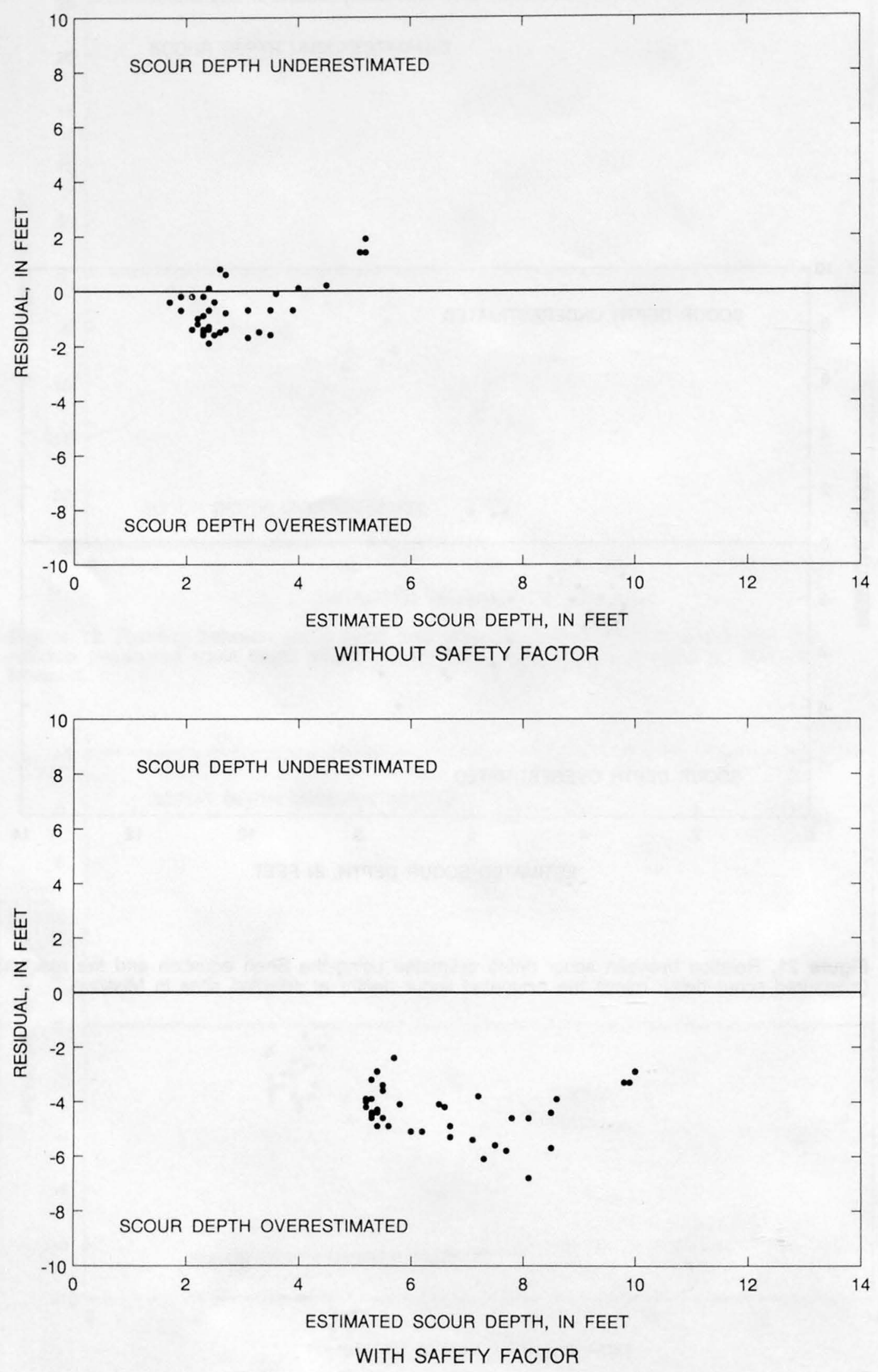

Figure 22. Relation between scour depth estimated using the Froehlich equation and the residual (measured scour depth minus the estimated scour depth) at selected sites in Missouri. 


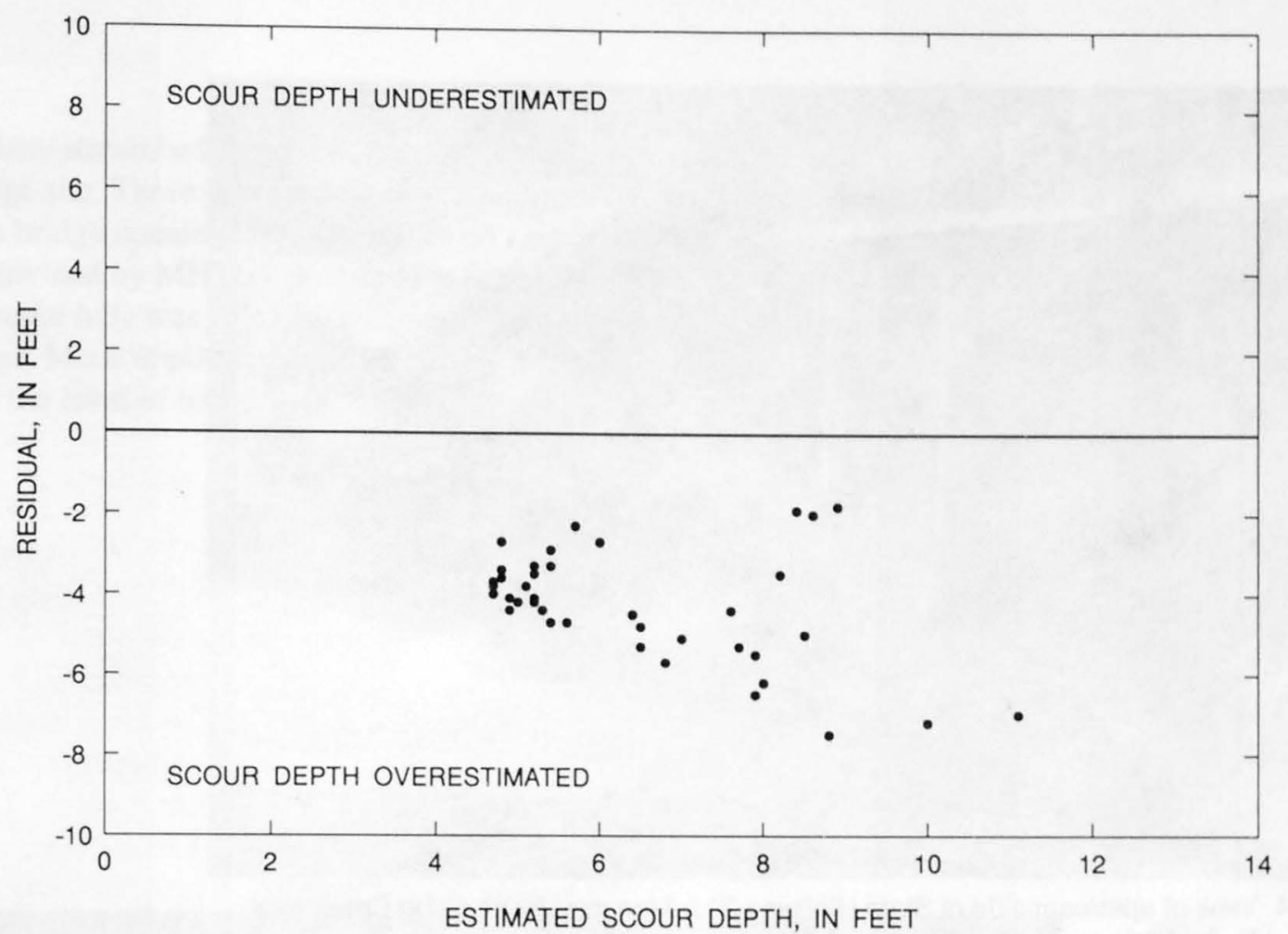

Figure 23. Relation between scour depth estimated using the Colorado State University equation and the residual (measured scour depth minus the estimated scour depth) at selected sites in Missouri.

\section{AN EXAMPLE OF A SCOUR-RELATED BRIDGE FAILURE}

Potential scour at bridges is a significant design and safety consideration. A bridge on State Highway 32 over North Cobb Creek (drainage area 52.5 square miles; fig. 1) was destroyed during a flood on May 26, 1990. Onsite conditions before and after this scour-related failure are shown in figures 24 and 25 . The failure of the more than 50-year old, 200-feet long, 8-span, pile-bent bridge was largely attributable to contraction scour. However, it is not known if or to what degree debris accumulation may have been a contributing factor. Bridge maintenance, including debris removal, had been accomplished by MHTD personnel a few days before the flood (Missouri Highway and Transportation Department, oral commun., 1990). Immediately after the failure, hydrologic, hydraulic, and geomorphic data were collected at the bridge site and adjacent areas.

This flood was the result of extremely intense rainfall of short duration over the entire drainage basin, averaging perhaps as much as 5 inches as shown by a "bucket survey" of rainfall amounts and information from local residents. Rainfall amounts from this survey ranged from a minimum of 3.0 inches to a maximum of 6.1 inches.

An estimated peak discharge of about 21,000 cubic feet per second occurred on May 26, 1990. This estimate is based on a transit-stadia onsite survey and use of Type II contracted-opening measurement methods (Matthai, 1967). This onsite survey was conducted on June 13 to 15, 1990. Flood high-water marks, streambed cross-sections, bridge and roadway details, and extensive field notes and photographs were obtained during this survey. Several streambed cross-sections were obtained upstream and downstream from the bridge site. Because the bridge, with the exception of the abutments, was completely destroyed, several assumptions were required in making the estimate of the peak discharge, including determining the time of the peak with respect to bridge failure and appropriate cross-sectional area of bridge opening. Based on this extensive onsite survey through analyses of streamflow profiles as indicated by surveyed high-water marks upstream and downstream from the highway, the bridge and substructure probably were destroyed and carried downstream from the bridge opening before the actual flood peak. The flood of May 26, 1990 (estimated discharge of 21,000 cubic feet per second), has a recurrence interval of approximately 50 years based on methods presented in a report by Becker (1986). 


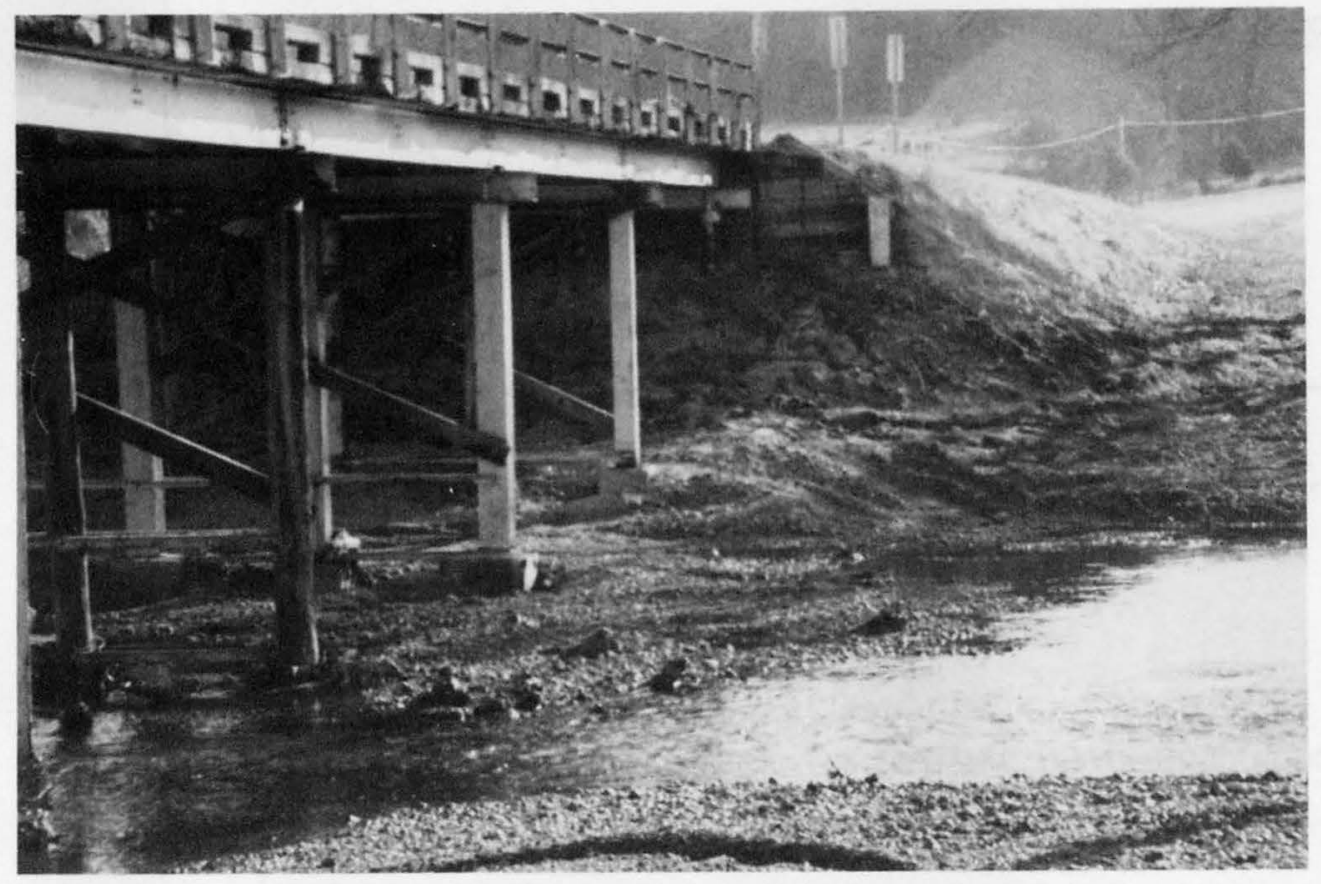

Figure 24. View of upstream side of State Highway 32 bridge over North Cobb Creek near Lebanon, Missouri (photograph of February 20, 1990, courtesy of the Missouri Highway and Transportation Department).

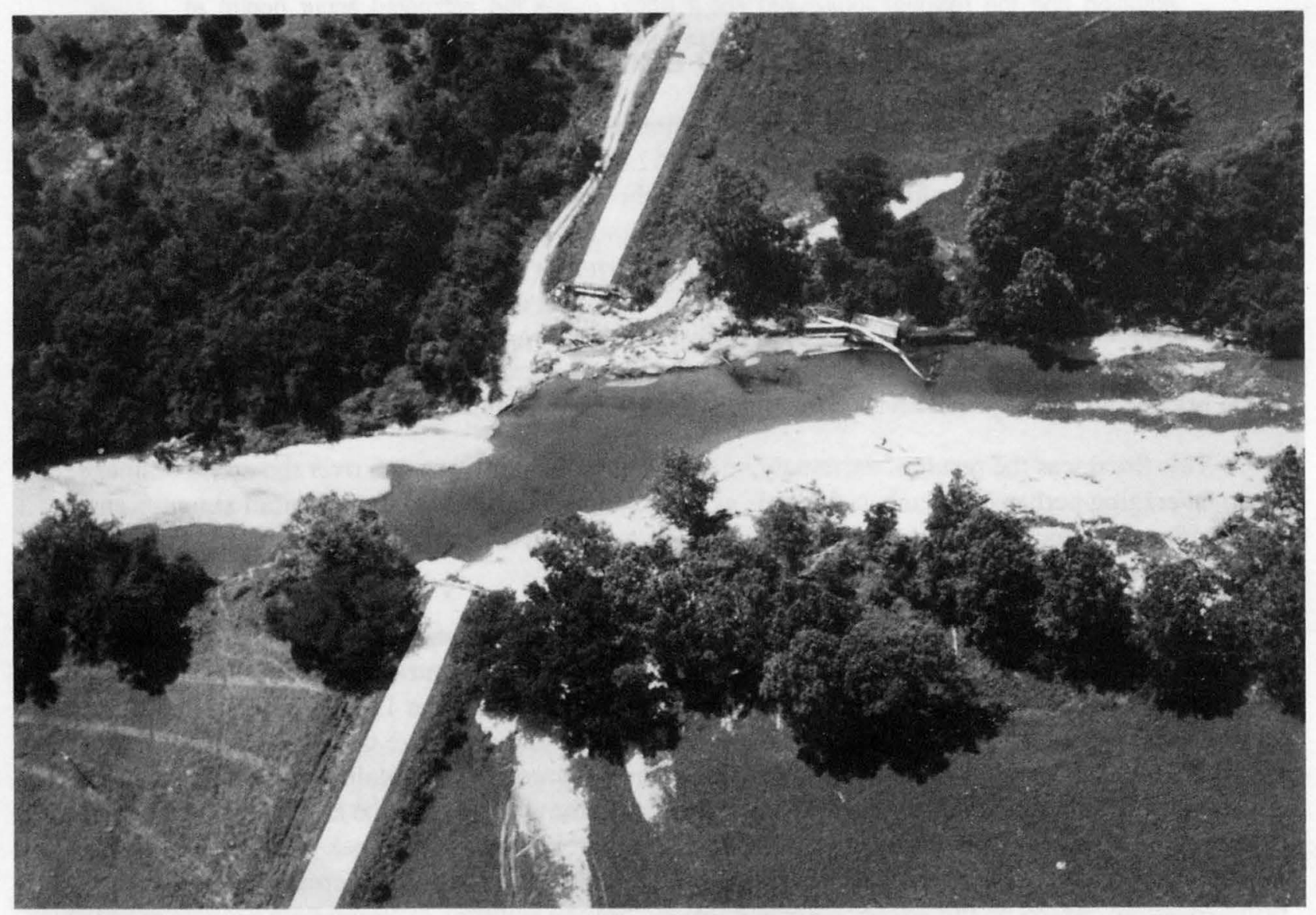

Figure 25. Aerial view of State Highway 32 crossing at North Cobb Creek, near Lebanon, Missouri, showing a scourrelated bridge failure that occurred during the flood of May 26, 1990 [direction of streamflow is from left to right; note sand and gravel banks deposited by flood downstream from highway (middle and bottom center of view); photograph of June 20, 1990]. 
Many streambed cross sections were obtained upstream from, at, and downstream from the highway and bridge site. These surveyed cross sections showed an extremely large scour hole at and downstream from the bridge opening (fig. 26). Based on surveyed cross sections, bridge plans dated November 14, 1932 (provided by MHTD), post-failure boring logs (provided by MHTD), and photographs (figs. 24 and 26) the scour hole was at least 6 feet deep and may have been as deep as 9 feet near the downstream side of the bridge. More importantly, onsite evidence indicated that scour to bedrock had occurred and that scour down to the level of or below the bottom of some of the timber-pile bents had occurred.

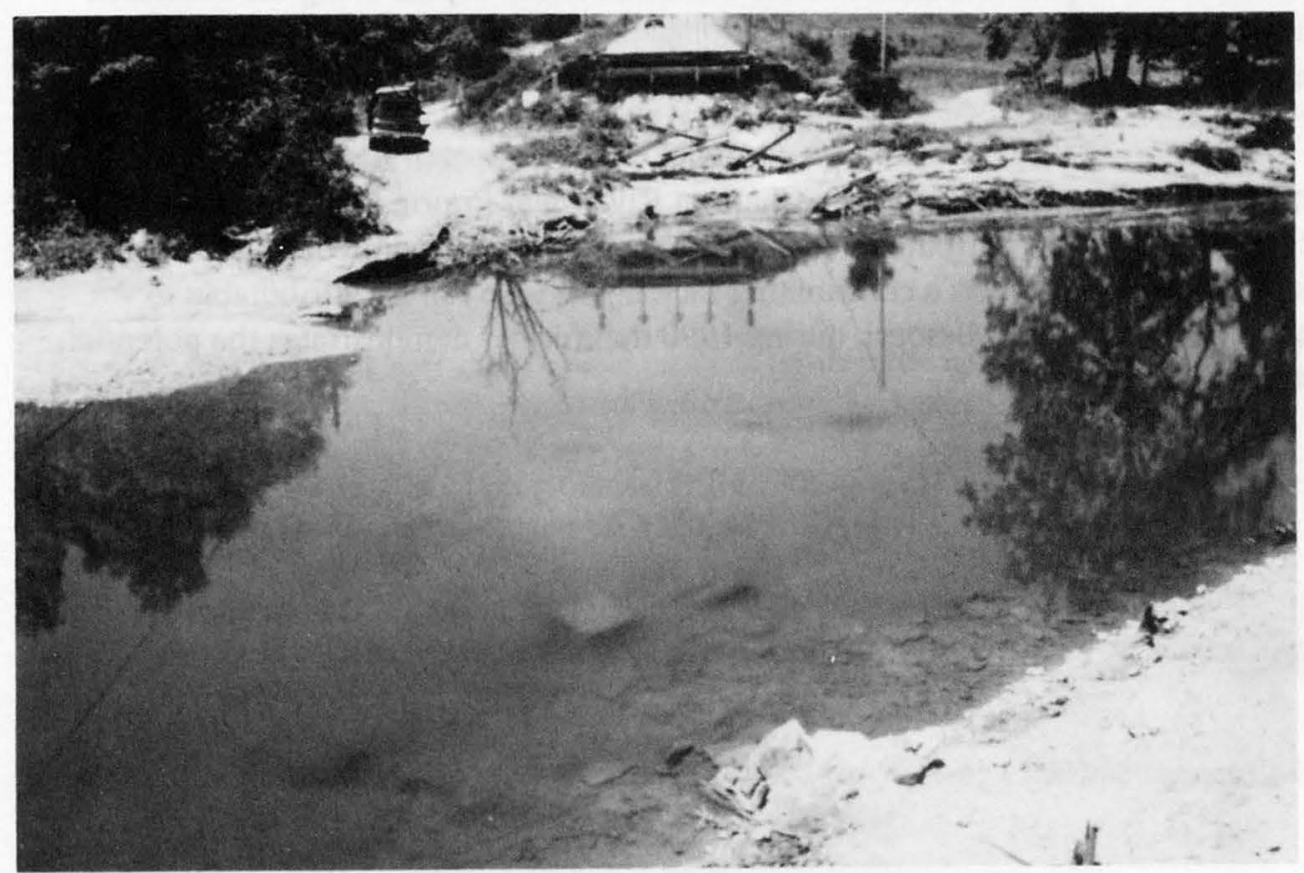

Figure 26. The large, deep scour hole formed during the flood of May 26, 1990, on North Cobb Creek near Lebanon, Missouri (photograph of June 14, 1990, is viewed from east abutment to west abutment of the destroyed bridge). 


\section{SUMMARY}

Local-scour data were collected at 10 sites in Missouri, 8 of which were at streamflow-gaging stations. Data collected were bridge-pier geometry, bed-material particle-size data, and hydraulic characteristics and cross-sectional data during selected flood events. Fathometer soundings, as well as manual soundings, were used to obtain streambed cross sections at upstream and downstream sides of bridges from which scour depth measurements at piers were determined. Data collected during this study and historical discharge measurement records produced 54 sets of scour data during 24 floods. The recurrence intervals of the floods ranged from less than 2 to greater than 50 years. Scour holes ranged from 0.5 to 7.1 feet deep.

Seven local-pier-scour equations were compared to determine their usefulness in estimating scour depths at the 10 study sites where scour was measured. The equations were those developed by Laursen, Larras, Chitale, Inglis-Poona II, Shen, Froehlich, and Colorado State University. For purposes of comparison, 39 of the most representative scour measurements and corresponding scour depth estimates were used. Froehlich's equation (without a safety factor) produced a median estimated scour depth statistically nearest to the median of the measured scour depth and was the equation that provided the "best fit" estimates. The residuals of estimated scour depths were plotted against the estimated scour depths to evaluate bias. Residuals for depths estimated using the seven equations indicated that all of these equations overestimated the measured scour depths. Residuals of the estimated scour depths using the Froehlich equation without a safety factor indicated the least significant bias in the estimated scour depths. Estimates from the Froehlich equation with a safety factor compare closely with estimates from the Colorado State University equation.

Total scour of about 19 feet was measured at the Chariton River near Prairie Hill during the extreme flooding of 1993. This scour was largely attributable to contraction scour; however, woody debris that partially blocked the bridge opening also was a contributing factor. A bridge failure attributable to contraction scour occurred near Lebanon, Missouri, during 1990 that further demonstrates the potential, detrimental effect of bridge scour. 


\section{SELECTED REFERENCES}

Becker, L.D.,1985, How the U.S. Geological Survey provides flood-estimating methods and conducts flood measurements [abs.]: Stormwater Conference, Jefferson City, Mo., 1985, Proceedings, p. 4-5.

1986, Techniques for estimating flood-peak discharges from urban basins in Missouri: U.S. Geological Survey Water-Resources Investigations Report 86-4322, 38 p.

1990, Simulation of flood hydrographs for small basins in Missouri: U.S. Geological Survey Water-Resources Investigations Report 90-4045, 40 p.

Butch, G.K., 1991, Measurement of bridge scour at selected sites in New York, excluding Long Island: U.S. Geological Survey Water-Resources Investigations Report 91-4083, 17 p.

Fenneman, N.M., 1938, Physiography of eastern United States: New York, McGraw-Hill, 714 p.

Froehlich, D.C., 1988, Analysis of on-site measurements of scour at piers, in Abt, S.R., and Gessler, Johannes, eds., Hydraulic engineering--Proceedings of the 1988 National Conference on Hydraulic Engineering: New York, American Society of Civil Engineers, p. 534-539.

Galay, V.J., 1983, Causes of river bed degradation: Water Resources Research, v. 19, no. 5, p. 1,057-1,090.

Gorin, S.R., and Haeni, F.P., 1989, Use of surface-geophysical methods to assess riverbed scour at bridge piers: U.S. Geological Survey Water-Resources Investigations Report 88-4212, 33 p.

Guy, H.P., 1969, Laboratory theory and methods for sediment analysis: U.S. Geological Survey Techniques of WaterResources Investigations, book 5, chapter $\mathrm{C} 1,58 \mathrm{p}$.

Guy, H.P., and Norman, V.W., 1970, Field methods for measurement of fluvial sediment: U.S. Geological Survey

Techniques of Water-Resources Investigations, book 3, chapter C2, 59 p.

Jarrett, R.D., and Boyle, J.M., 1986, Pilot study for collection of bridge-scour data: U.S. Geological Survey WaterResources Investigations Report 86-4030, 46 p.

Lagasse, P.F., Shall, J.D., Johnson, F., Richardson, E.V., Richardson, J.R., and Chang, F., 1991, Stream stability at highway structures: Washington, D.C., Department of Transportation, Hydraulic Engineering Circular No. 20, FHWA-IP-90-014.

Landers, M.N., 1991, A bridge scour measurement data base system: Fifth Interagency Sedimentation Conference, Las Vegas, Nev., 1991, Proceedings, v. 2, p. 121-126.

Landers, M.N., and Mueller, D.S., 1993, Reference surfaces for bridge scour depths, in Shen, H.W., Su, S.T., and Wen Feng, eds., Hydraulic Engineering '93: Hydraulics Division of the American Society of Civil Engineers Conference, San Francisco, Calif., Proceedings, 1993, p. 2,075-2,080.

Landers, M.N., and Trent, R.E., 1991, A national bridge scour data collection program, in Hydraulic Engineering 1991, American Society of Civil Engineers, Nashville, Tenn., p. 221-226.

Laursen, E.M., 1962, Scour at bridge crossings: Transactions of the American Society of Civil Engineers, v. 127, Part I, no. 3294, p. 166-209.

Matthai, H.F., 1967, Measurement of peak discharge at width contractions by indirect methods: U.S. Geological Survey Techniques of Water-Resources Investigations, book 3, chapter A4, 44 p. 
Mueller, D.S., Miller, R.L. and Wilson, J.T., 1994, Historical and potential scour around bridge piers and abutments of selected stream crossings in Indiana: U.S. Geological Survey Water-Resources Investigations Report 93-4066, $109 \mathrm{p}$.

Parrett, Charles, Melcher, N.B., and James, Jr., R.W., 1993, Flood discharges in the upper Mississippi River Basin, 1993, in Floods in the upper Mississippi River Basin, 1993: U.S. Geological Survey Circular 1120-A, 14 p.

Rantz, S.E., and others, 1982, Measurement and computation of streamflow--Volume 1, Measurement of stage and discharge: U.S. Geological Survey Water-Supply Paper 2175, 284 p.

Richardson, E.V., Harrison, L.J., and Davis, S.R., 1991, Evaluating scour at bridges: Washington, D.C., Department of Transportation, Hydraulic Engineering Circular No. 18, FHWA-IP-90-017.

Simon, Andrew, and Outlaw, G.S., 1989, Evaluation, modeling, and mapping of potential bridge-scour, west Tennessee: Proceedings of the October 1989 Bridge Scour Symposium, Federal Highway Administration, McLean, Va., p. 112-129.

Southard, R.E., 1989, A mobile scour monitoring system for use at bridges: Proceedings of the October 1989 Bridge Scour Symposium, Federal Highway Administration, McLean, Va., p. 175-181.

1992, Scour around bridge piers on streams in Arkansas: U.S. Geological Survey Water-Resources Investigations Report 92-4126, 29 p. 
